\title{
Hidden symmetries of Eisenhart-Duval lift metrics and the Dirac equation with flux
}

\author{
Marco Cariglia* \\ Departamento de Física, Universidade Federal de Ouro Preto, ICEB, Campus Morro do Cruzeiro, \\ Morro do Cruzeiro, 35400-000 Ouro Preto, MG, Brasil
}

(Received 21 June 2012; published 26 October 2012)

\begin{abstract}
The Eisenhart-Duval lift allows embedding nonrelativistic theories into a Lorentzian geometrical setting. In this paper we study the lift from the point of view of the Dirac equation and its hidden symmetries. We show that dimensional reduction of the Dirac equation for the Eisenhart-Duval metric in general gives rise to the nonrelativistic Lévy-Leblond equation in lower dimension. We study in detail in which specific cases the lower dimensional limit is given by the Dirac equation, with scalar and vector flux, and the relation between lift, reduction, and the hidden symmetries of the Dirac equation. While there is a precise correspondence in the case of the lower dimensional massive Dirac equation with no flux, we find that for generic fluxes it is not possible to lift or reduce all solutions and hidden symmetries. As a byproduct of this analysis, we construct new Lorentzian metrics with special tensors by lifting Killing-Yano and closed conformal Killing-Yano tensors and describe the general conformal Killing-Yano tensor of the Eisenhart-Duval lift metrics in terms of lower dimensional forms. Last, we show how, by dimensionally reducing the higher dimensional operators of the massless Dirac equation that are associated with shared hidden symmetries, it is possible to recover hidden symmetry operators for the Dirac equation with flux.

DOI: 10.1103/PhysRevD.86.084050

PACS numbers: 04.50.- $\mathrm{h}, 03.65 . P m, 11.30 .-\mathrm{j}$
\end{abstract}

\section{INTRODUCTION}

The Eisenhart-Duval lift of a Riemannian metric [1] is an example of geometrization of interactions. The dynamics of a classical physical system, described by a Riemannian metric $h_{\mu \nu}$ in $n$ dimensions and in the presence of a scalar potential $V$ and a vector potential $A_{\mu}$, is shown to be equivalent to geodesics in a Lorentzian spacetime of dimension $n+2$. This geometrical idea has been historically introduced by Eisenhart in Ref. [1]; however, to our knowledge it took a number of years after the same idea was independently rediscovered in Ref. [2], from there prompting further work, among which are Refs. [3,4]. Over time it has found a number of applications, among which one can mention the following, nonexhaustive, examples: providing a relativistic framework to study nonrelativistic physics, as the free Schrödinger equation in $n$ dimensions and with metric $g$ can be written in the lifted geometry as the free, massless Klein-Gordon equation [5]; simplifying the study of symmetries of a Hamiltonian system by looking at geodesic Hamiltonians [6,7]; building new Lorentzian $p p$-wave metrics solutions of the Einstein-Maxwell equations $[4,8]$; and studying from a geometrical point of view dynamical systems as diverse as protein folding [9], rare gas crystals [10], and chaotic gravitational $N$-body systems [11].

On a separate account, there has been much recent activity in the study of hidden symmetries of physical systems. The interest has increased for two main reasons. First, it has been discovered that a number of hidden symmetries are related to separation of variables for equations of physics related to different spin and of either

\footnotetext{
*marco@iceb.ufop.br
}

classical or quantum nature: the geodesic equation [12], the Hamilton-Jacobi and Klein-Gordon equation [13,14], the Dirac equation [15,16], electromagnetic perturbations in $n=5$ dimensions [17], and linearized gravitational perturbations [17-21]. Such separation has been achieved for Kerr-NUT-(A)dS spacetimes [22], which are higherdimensional generalizations of the Kerr metric with cosmological constant and NUT charge. For these spacetimes it has been proven that a principal conformal Killing-Yano tensor is present [23] and that from it all the geometrical structure and further hidden symmetries follow [24]. It is also possible to show that the theory of the worldline supersymmetric spinning particle in these spacetimes admits a number of nontrivial supercharges that make its bosonic sector integrable [25]. In general, see Refs. [26-28] for an extensive review of hidden symmetries in the framework of gravitational systems. The second reason for the recent activity is the fact that several new examples of spacetimes with nontrivial hidden symmetries of higher order have been found, in many cases using the Eisenhart-Duval lift procedure applied to integrable systems such as the Goryachev-Chaplygin top, the Kovalevskaya top, and the Calogero model [29-31].

In this paper we look at the Eisenhart-Duval lift procedure from the point of view of hidden symmetries of the Dirac equation and of conformal Killing-Yano tensors (CKY). There is a number of reasons why this is meaningful. First, if it is possible to perform the Eisenhart-Duval lift of a known CKY tensor, then this opens the possibility to create new Lorentzian metrics with CKY tensors. To this extent it is useful to note that a classification of higher dimensional spacetimes with CKY tensors has only been completed in the case of rank 2 closed tensors and 
Riemannian signature with and without torsion [32-35]. In this paper we show how to perform such a lift under appropriate conditions, thus presenting new Lorentzian metrics with CKY tensors. Second, since the EisenhartDuval lift links a higher dimensional dynamics in the absence of forces other than gravity to that of a lower dimensional system in the presence of scalar and vector potential, there is the possibility to establish a link between the higher dimensional (massless) Dirac equation and a Dirac equation in lower dimension with scalar and vector flux. In the paper we show concretely how to perform the dimensional reduction of the higher dimensional Dirac equation and obtain the lower dimensional Dirac equation with flux and, its inverse operation, oxidation. This is of interest in itself and more so since we are able to show a geometrical link between hidden symmetry operators of the free higher dimensional Dirac equation, which are given in terms of CKY tensors [36,37], and the recently discussed hidden symmetry operators of the Dirac equation with flux [38]. While analyzing the hidden symmetries of the higher dimensional and lower dimensional theories, we find the nontrivial result that for generic fluxes each of the two theories can have hidden symmetries that are not present in the other. It is worth noticing that this phenomenon is not present when we relate the massive lower dimensional Dirac equation with the other fluxes turned off and the massless higher dimensional Dirac equation. For those hidden symmetries that are common to the two theories, we can perform dimensional reduction and find the symmetry operators with flux discussed in Ref. [38]. Under the hypothesis that symmetry operators linear in momenta for the lower dimensional theory cannot be lifted to symmetry operators in higher dimension of order $>1$, we interpret this result as meaning that the two theories differ as the level of phase space dynamics, which is different from what happens in the case of a scalar particle. A third reason to study CKY tensors for Eisenhart-Duval lift metrics is that it is possible to characterize the higher dimensional CKY equation completely in terms of lower dimensional forms. We do this and obtain equations in lower dimensions that generalize the CKY equation and implicitly classify the most general higher dimensional CKY tensor.

The rest of the paper is organized as follows. In Sec. II we introduce useful notation and basic notions about hidden symmetries. Section III is devoted to the Eisenhart-Duval lift. We discuss the geometrical lift and the dynamics of a scalar particle and its hidden symmetries. Finally, we show how to lift lower dimensional CKY tensors and the fact that there are restrictions to this procedure. We also classify the most general higher dimensional CKY tensor in terms of lower dimensional forms. Section IV is devoted to analyzing the dimensional reduction of the Dirac equation in higher dimension. We show that in general one can recover in lower dimension the nonrelativistic Lévy-Leblond equation. In addition, it is also possible in some cases to recover the lower dimensional Dirac equation with flux using the higher dimensional massless Dirac equation plus a nontrivial projection. Such a projection is responsible for the fact that not all higher dimensional linear symmetry operators can be dimensionally reduced: for a specific class of symmetry operators we show in detail when this can be done and obtain a subset of the linear symmetry operators of the Dirac equation with flux discussed in Ref. [38]. We also use the Dirac equation to gain insight on the earlier finding that not all lower dimensional CKY tensors can be lifted. Section V presents some examples, and finally Sec. is devoted to conclusions and final remarks. In the Appendices we discuss useful identities for the metric, Hodge duality, and differentiation of forms, and we present the full set of higher dimensional CKY equation in terms of equations for lower dimensional forms.

\section{PRELIMINARIES}

\section{A. Notation}

We start with a Riemannian metric $g$ defined on an $n$-dimensional manifold $\mathcal{M}$. Its Eisenhart-Duval lift will be an $(n+2)$-dimensional manifold $\hat{\mathcal{M}}$, which is a bundle over $\mathcal{M}$ on which a Lorentzian metric $\hat{g}$ is defined. In general, $(n+2)$-dimensional quantities will be denoted with a hat symbol, so, for example, if $f$ is a $p$ form on $\mathcal{M}$, then its natural embedding in $\hat{\mathcal{M}}$ will be denoted by $\hat{f}$; if $D$ is the Dirac operator on $\mathcal{M}$, then $\hat{D}$ will be the Dirac operator on $\hat{\mathcal{M}}$; and so on. Indices $\mu, \nu, \ldots$, from the lowercase Greek alphabet represent spacetime indices on $\mathcal{M}$, while $M, N, \ldots$, from the uppercase Latin alphabet represent spacetime indices on $\hat{\mathcal{M}}$. Local coordinates used for $\mathcal{M}$ are $\left\{x^{\mu}\right\}$, and for the lift we introduce new variables $v, t$ so that $\left\{\hat{x}^{M}\right\}=\left\{v, t,\left\{x^{\mu}\right\}\right\}$ are local coordinates on $\hat{\mathcal{M}}$. When we work with the Dirac equation and Gamma matrices it is convenient to use locally flat indices: we will use $a=1, \ldots, n$ for $\mathcal{M}$ and $A=+,-, 1, \ldots, n$ for $\hat{\mathcal{M}}$. Vielbein forms on $\mathcal{M}$ are indicated as $e^{a}=e_{\mu}^{a} d x^{\mu}$ and, analogously, on $\hat{\mathcal{M}}$ as $\hat{e}^{A}=\hat{e}_{M}^{A} d \hat{x}^{M}$. Inverse vielbeins are written as $E_{a}^{\mu}$ and $\hat{E}_{A}^{M}$, respectively. $\nabla$ always means the appropriate covariant derivative, acting on either tensors, forms or spinors.

The notation we use to describe differential forms is the following: we display formulas valid on $\mathcal{M}$, and similar formulas hold for $\hat{\mathcal{M}}$. Let $\left\{d x^{\mu}\right\}$ be a coordinate basis for 1 forms and $\left\{\partial_{\mu}\right\}$ for vectors. The exterior algebra is $\Omega(\mathcal{M})=\bigoplus_{p=0}^{n} \Omega^{p}(\mathcal{M})$. Given a $p$ form $\omega=$ $\frac{1}{p !} \omega_{\mu_{1} \ldots \mu_{p}} d x^{\mu_{1}} \ldots d x^{\mu_{p}} \in \Omega^{p}(\mathcal{M})$ and a vector $v$, the inner derivative of $\omega$ relative to $v$, or hook operation, is a $(p-1)$ form $v\lrcorner \omega$ with components given by

$$
(v\lrcorner \omega)_{\mu_{1} \ldots \mu_{p-1}}=v^{\lambda} \omega_{\lambda \mu_{1} \ldots \mu_{p-1}} .
$$


Given a vector $v=v^{\mu} \partial_{\mu}$, there is a canonical form associated with it using the metric to lower the component indices, $v=v_{\mu} d x^{\mu}$, and similarly given a 1 form $\lambda=$ $\lambda_{\mu} d x^{\mu}$, there is a vector $\lambda^{\#}=\lambda^{\mu} \partial_{\mu}$. Such an operation is also called musical isomorphism. Given a vielbein basis for 1 forms $\left\{e^{a}\right\}$, then the vectors $X^{a}=\left(e^{a}\right)^{\#}$ are a basis for the tangent space of $\mathcal{M}$ and satisfy $\left(X^{a}\right)^{\mu}\left(X^{b}\right)_{\mu}=\eta^{a b}$, where $\eta$ is the unit matrix for $\mathcal{M}$ (and $\hat{\eta}$ is the Minkowski metric for $\hat{\mathcal{M}}$ ). Then the differential and codifferential of a form $\omega$ can be written as

$$
\left.d \omega=e^{a} \wedge \nabla_{a} \omega, \quad \delta \omega=-X^{a}\right\lrcorner \nabla_{a} \omega .
$$

An inhomogeneous form $\omega$ can be written as a sum of homogeneous $p$ forms

$$
\omega=\sum_{p=0} \omega^{(p)}
$$

We define the degree operator $\pi$ and parity operator $\eta$, which act as

$$
\pi \omega=\sum_{p=0} p \omega^{(p)}, \quad \eta \omega=\sum_{p=0}(-1)^{p} \omega^{(p)} .
$$

For $\alpha, \beta$, a $p$, and, respectively, $q$ form, we define the contracted wedge product recursively by

$$
\begin{aligned}
& \alpha \wedge \beta=\alpha \wedge \beta \\
& \left.\left.\alpha \wedge \beta=\left(X_{a}\right\lrcorner \alpha\right) \underset{k-1}{\wedge}\left(X^{a}\right\lrcorner \beta\right) \quad(k \geq 1), \\
& \alpha \wedge \beta=0 \quad(k<0) \text {. }
\end{aligned}
$$

The contracted wedge product satisfies the identities

$$
\begin{aligned}
& \left.e^{a} \wedge\left[\left(X_{a}\right\lrcorner \alpha\right) \underset{m}{\wedge} \beta\right]=(-1)^{m}[(\pi-m) \alpha] \underset{m}{\wedge} \beta, \\
& \left.e^{a} \wedge\left[\alpha{ }_{m}\left(X_{a}\right\lrcorner \beta\right)\right]=\left[(-1)^{\pi} \alpha\right]{\underset{m}{\wedge}}_{\wedge}[(\pi-m) \beta] .
\end{aligned}
$$

When dealing with the Dirac equation, we use the following isomorphism $\gamma_{*}$ between $\Omega(\mathcal{M})$ and the Clifford bundle:

$$
\begin{aligned}
f & =\frac{1}{p !} f_{\mu_{1} \ldots \mu_{p}} d x^{\mu_{1}} \ldots d x^{\mu_{p}} \mapsto \\
\gamma_{*}(f) & =\frac{1}{p !} f_{\mu_{1} \ldots \mu_{p}} \Gamma^{\mu_{1} \ldots \mu_{p}} .
\end{aligned}
$$

Here $\left\{\Gamma^{\mu}\right\}$ are the Gamma matrices, satisfying the standard relation $\Gamma^{\mu} \Gamma^{\nu}+\Gamma^{\nu} \Gamma^{\mu}=2 g^{\mu \nu}, \quad \Gamma^{\mu_{1} \ldots \mu_{p}}=\Gamma^{\left[\mu_{1}\right.} \ldots \Gamma^{\left.\mu_{p}\right]}$, and the equation above straightforwardly generalizes to the case of an inhomogeneous form. Any time the context makes it clear that quantities refer to the Clifford bundle, we will write $f$ instead of $\gamma_{*}(f)$. For example, under these conditions the Dirac operator is written as $D=e^{a} \nabla_{a}$. The product of two Clifford bundle forms can be reexpressed in terms of contracted wedge products using the Gamma matrix algebra. Let $\alpha \in \Omega^{p}(M), \beta \in \Omega^{q}(M)$, and $p \leq q$. Then the Clifford product expands as

$$
\alpha \beta=\sum_{m=0}^{p} \frac{(-1)^{m(p-m)+[m / 2]}}{m !} \alpha \underset{m}{\wedge} \beta
$$

and

$$
\beta \alpha=(-1)^{p q} \sum_{m=0}^{p} \frac{(-1)^{m(p-m+1)+[m / 2]}}{m !} \alpha{ }_{m} \beta .
$$

\section{B. Basics of hidden symmetries}

Hidden symmetries of a Hamiltonian physical system are associated with conserved quantities of the dynamics that are polynomial in the momenta. If the system is classical by momenta, we mean the variables $p_{\mu}$ canonically conjugated to the position variables $x^{\mu}$, and if the system is quantum mechanical, we mean the appropriate operators. When the spacetime admits a Killing vector $K$, then the conserved quantity is of order one in the momenta, and vice versa, if there is such a quantity, it can be written in terms of a Killing vector and its derivatives. Of particular importance are the following two classes of special tensors.

Killing-Stäckel tensors (KS) are symmetric tensors $K^{\mu_{1} \ldots \mu_{p}}=K^{\left(\mu_{1} \ldots \mu_{p}\right)}$ satisfying the differential equation

$$
\nabla^{(\lambda} K^{\left.\mu_{1} \ldots \mu_{p}\right)}=0 .
$$

They generate conserved quantities

$$
C_{K}=K^{\mu_{1} \ldots \mu_{p}} p_{\mu_{1}} \ldots p_{\mu_{p}}
$$

for the theory of the classical free scalar particle in curved space. A well-known example is given by Carter's constant for the Kerr metric, and Carter-like constants for curved backgrounds keep being discovered in recent research [39]. Quantum mechanically, the corresponding operators in the case of rank 2 are given by

$$
\mathcal{K}=\nabla_{\mu}\left[K^{\mu \nu} \nabla_{\nu}\right]
$$

but these do not always generate conserved quantities, as the commutator $\left[\mathcal{K}, \nabla_{\mu} \nabla^{\mu}\right]$ is given by an appropriate contraction of the Ricci tensor with $K$ [40]. Failure of the commutator to close on zero indicates that the classical symmetry is gravitationally anomalous. If the spacetime is special, for example, in the case of the Kerr-NUT-(A)dS spacetime, then the anomaly vanishes. Another special case when the anomaly vanishes is when the KS tensor can be written as the square of a Killing-Yano tensor, which will be defined below. In the case of rank $2 \mathrm{KS}$ tensors, the theory of the supersymmetric spinning particle admits a superfield that is the generalization of the phase space function $K^{\mu \nu} p_{\mu} p_{\nu}$, a candidate conserved quantity that is also supersymmetric. In this case, too, in general there is an anomaly and the superfield is not supersymmetric nor conserved, but for Kerr-NUT-(A)dS spacetimes the anomaly vanishes [25]. Finally, it is worth noticing that a Killing vector is a Killing-Stäckel tensor of rank 1. 
Conformal Killing-Yano tensors are forms $\omega_{\mu_{1} \ldots \mu_{p}}=$ $\omega_{\left[\mu_{1} \ldots \mu_{p}\right]}$ such that

$$
\begin{aligned}
\nabla_{\lambda} \omega_{\mu_{1} \ldots \mu_{p}}= & \nabla_{[\lambda} \omega_{\left.\mu_{1} \ldots \mu_{p}\right]} \\
& +\frac{p}{D-p+1} g_{\lambda\left[\mu_{1}\right.} \nabla^{\rho} \omega_{\left.|\rho| \mu_{2} \ldots \mu_{p}\right]},
\end{aligned}
$$

or equivalently without using components

$$
\left.\nabla_{X} \omega=\frac{1}{\pi+1} X\right\lrcorner d \omega-\frac{1}{n-\pi+1} X^{b} \wedge \delta \omega,
$$

for any vector $X$. This formula generalizes automatically to the case of inhomogeneous forms. When $\omega$ is coclosed, $\delta \omega=0, \omega$ is a Killing-Yano form (KY), and when it is closed, $d \omega=0$, it is a closed conformal Killing-Yano form (CCKY). Equation (2.14) is invariant under Hodge duality, interchanging $\mathrm{KY}$ and CCKY tensors. Benn, Charlton, and Kress [36,37] have shown the important result that, in all dimensions $n$ and arbitrary signature, first-order symmetry operators of the massless Dirac operator are in one-to-one correspondence with CKY forms. Specifically, if $S$ is an operator satisfying $D S=R D$ for some operator $R$, then $S$ is given by

$$
S=S_{\omega}+\alpha D,
$$

where $\alpha$ is an arbitrary inhomogeneous form, and $S_{\omega}$, given in terms of an inhomogeneous CKY form $\omega$ obeying (2.14), is

$$
\left.S_{\omega}=X^{a}\right\lrcorner \omega \nabla_{a}+\frac{\pi-1}{2 \pi} d \omega-\frac{n-\pi-1}{2(n-\pi)} \delta \omega .
$$

Then $S_{\omega}$ obeys

$\left[\left|D, S_{\omega}\right|\right] \equiv D S_{\omega}-\left(\eta S_{\omega}\right) D=-\left(\frac{\eta}{n-\pi} \delta \omega\right) D$.

The freedom of adding an arbitrary form $\alpha$ is unavoidable. It can also be shown that if $\omega$ is a CCKY form, then the operator

$$
S_{\omega}=e^{a} \wedge \omega \nabla_{a}-\frac{n-\pi-1}{2(n-\pi)} \delta \omega
$$

either commutes or anticommutes with the Dirac operator, depending whether $\omega$ is even or odd [41].

Similar results hold in the case of the spinning particle; see, for example, Ref. [42] for the discussion of KY tensors. For the Kerr-NUT-(A)dS metrics it is possible to show that there exist $n$ independent such operators, as many as the number of dimensions (one of them being $D$ itself), that they all mutually commute and that this explains the separation of variables for the Dirac equation in these metrics [16,41].

If a spacetime admits Killing spinors, with or without torsion, then these can be used to build CKY tensors [43-45]. The Dirac equation with skew-symmetric torsion has been discussed in Ref. [46]. The link between CKY tensors and $G$ structures has been discussed in Refs. [47-49]. For application of the idea of hidden symmetries to other systems see, for example, Refs. [50,51].

\section{THE EISENHART-DUVAL LIFT}

\section{A. The geometric lift}

In this paper we will consider the Eisenhart-Duval lift in the time independent case. Let $\mathcal{M}$ be an $n$-dimensional Riemannian spacetime, with metric

$$
g=g_{\mu \nu}(x) d x^{\mu} d x^{\nu} .
$$

On $\mathcal{M}$ we can consider the classical theory of a particle of mass $m$ and electric charge $e$, interacting with a position dependent potential $V(x)$ and with a stationary electromagnetic field with vector potential $A_{\mu}(x)$, introducing the Hamiltonian

$$
\mathcal{H}=\frac{1}{2 m} g^{\mu \nu}\left(p_{\mu}-e A_{\mu}\right)\left(p_{\nu}-e A_{\nu}\right)+V(x),
$$

where $p_{\mu}$ is the canonical momentum. The Hamiltonian function written above is not explicitly invariant under a gauge transformation of the vector potential, but the full theory is. What happens is that under a gauge transformation the canonical momenta change as well as the vector potential, generating a canonical transformation. As a result the Hamiltonian equations of motion are not explicitly gauge invariant, but the theory, in fact, is. It is possible to introduce gauge invariant momenta $P_{\mu}=p_{\mu}-e A_{\mu}$ while at the same time modifying the Poisson brackets and recognizing that $P$ acts as a $U(1)$ covariant derivative; see Refs. [42,52-57]. This is not needed to the extent of the calculations done in this paper, and we will work with the canonical $p_{\mu}$ variables.

It is a result by Eisenhart [1] that the Hamiltonian (3.1) can be obtained by reduction from the following Hamiltonian in $(n+2)$ dimensions:

$$
\begin{aligned}
\hat{\mathcal{H}}= & \hat{g}^{M N} \hat{p}_{M} \hat{p}_{N} \\
= & g^{\mu \nu}\left(p_{\mu}-\frac{e}{m} A_{\mu} p_{v}\right)\left(p_{\nu}-\frac{e}{m} A_{\nu} p_{v}\right) \\
& +2 p_{v} p_{t}+\frac{2}{m} V p_{v}^{2},
\end{aligned}
$$

where $\hat{p}_{M}=\left(p_{v}, p_{t}, p_{\mu_{1}}, \ldots, p_{\mu_{n}}\right) . \hat{\mathcal{H}}$ describes the motion of a massless particle in the higher dimensional Lorentzian metric

$$
\begin{aligned}
\hat{g} & =\hat{g}_{M N} d \hat{x}^{M} d \hat{x}^{N} \\
& =g_{\mu \nu} d x^{\mu} d x^{\nu}+\frac{2 e}{m} A_{\mu} d x^{\mu} d t+2 d t d v-\frac{2}{m} V d t^{2} .
\end{aligned}
$$

To see this, consider the coordinate $v$, generated by the covariantly constant Killing vector $\xi=\partial / \partial v$, which is 
conjugate to $p_{v} . p_{v}$ is constant along a solution of the equations of motion. If we specialize to a null solution with $\hat{\mathcal{H}}=0$ and choose $p_{v}=m$, then we have

$$
0=\hat{\mathcal{H}}=g^{\mu \nu}\left(p_{\mu}-e A_{\mu}\right)\left(p_{\nu}-e A_{\nu}\right)+2 m p_{t}+2 m V,
$$

or equivalently

$$
p_{t}=-\mathcal{H} \text {. }
$$

This means we can identify $\mathcal{H}$, generator of time translations in the $n$-dimensional system, with $-p_{t}$ in the $(n+2)$-dimensional one, which generates translations along $-\partial / \partial t$. Expressions for the vielbeins of the metric (3.4), as well as the dual vector base, the Levi-Civita connection, and spin connection components can be found in Appendix A.

Geometrically we can describe $\hat{\mathcal{M}}$ as a bundle over $\mathcal{M}$, with projection $\Pi:\left(t, v, x^{\mu}\right) \mapsto x^{\mu}$. Then if $f$ is a $p$ form defined on $\mathcal{M}$, its pullback on $\hat{\mathcal{M}}$ under the map $\Pi^{*}$ is a $p$ form $\hat{f}$ on $\hat{\mathcal{M}}$.

As seen above, null geodesics on $\hat{\mathcal{M}}$ relative to $\hat{g}$ generate massive geodesics on $\mathcal{M}$ relative to $g$. It is, in fact, possible to do more: given a generic conserved quantity for the motion on $\mathcal{M}$ that is a nonhomogeneous polynomial in momenta, in other words a hidden symmetry, this can be lifted to an appropriate hidden symmetry on $\hat{\mathcal{M}}$ that is homogeneous in momenta and that therefore is associated with a Killing tensor. The Poisson algebra on $\mathcal{M}$ of conserved charges for the original motion then is the same as the Schouten-Nijenhuis algebra of the Killing tensors associated with lifted conserved charges [29]. This means that the dynamical evolution on $\mathcal{M}$ as described in full phase space can be embedded in the higher dimensional phase space. As we will see, this does not happen in the case of the Dirac equation, where, in general, it will not be possible to lift all hidden symmetries from $\mathcal{M}$ to $\hat{\mathcal{M}}$. This corresponds to the fact that when performing the dimensional reduction of the Dirac equation on $\hat{\mathcal{M}}$ a nontrivial projection is required in phase space in order to recover the Dirac equation with $V$ and $A$ flux on $\mathcal{M}$. This projection is not compatible with all hidden symmetry transformations.

\section{B. Lift of conformal Killing-Yano forms}

In this section we consider the CKY equation on $\hat{\mathcal{M}}$ :

$$
\left.\hat{\nabla}_{\hat{X}} \hat{f}=\frac{1}{\pi+1} \hat{X}\right\lrcorner \hat{d} \hat{f}-\frac{1}{(n+2)-\pi+1} \hat{X}^{b} \wedge \hat{\delta} \hat{f},
$$

$\forall \hat{X}$ vector. We will specialize to a homogeneous form $\hat{f}$, since any nonhomogeneous CKY form can be split into a sum of homogeneous CKY forms. CKY forms are the appropriate forms to look for, since in $(n+2)$ dimensions we are focusing on null geodesics that are in correspondence with geodesics on the base manifold $\mathcal{M}$, and since they generate symmetries of the massless Dirac equation.

Before studying the general case we begin with four simplified ansätze for the higher dimensional CKY form. Given a $p$ form $f=f(x)$ living on the base manifold $\mathcal{M}$, we can build the following higher dimensional forms:

$$
\begin{aligned}
& \hat{f}_{1}=f, \\
& \hat{f}_{2}=e^{+} \wedge f, \\
& \hat{f}_{3}=e^{-} \wedge f, \\
& \hat{f}_{4}=e^{+} \wedge e^{-} \wedge f,
\end{aligned}
$$

where by writing on the right-hand side $f$ instead of $\hat{f}$ for a form on $\hat{M}$ we are performing a slight abuse of notation with the purpose of indicating that $f$ represents the canonical embedding in $\hat{\mathcal{M}}$ of a form originally defined on $\mathcal{M}$. Since in principle each of the four forms above can be multiplied times a function of the $t$ and $v$ variables, we allow from the beginning for a $p$ form $\hat{f}_{i}=\hat{f}_{i}(v, t, x)$ that can have $v, t$ dependence. Hodge duality in $\hat{\mathcal{M}}$ maps a form of type $\hat{f}_{1}$ into one of type $\hat{f}_{4}$-after allowing for $f \rightarrow * \mathcal{M} f$. Similarly, it relates the forms $\hat{f}_{2}$ and $\hat{f}_{3}$ to themselves.

We will first study the conditions under which these four ansätze generate CKY tensors on $\hat{\mathcal{M}}$. After that we will study the equations for the general CKY tensor. Two main findings are worth noticing. One is that in case of Eqs. (3.8) and (3.11) it is possible to generate KY and CCKY, respectively, tensors on $\hat{\mathcal{M}}$ when $f$ is $\mathrm{KY}$ and CCKY, on $\mathcal{M}$. By doing this we can construct new examples of Lorentzian metrics with CKY tensors by lifting known CKY tensors in Riemannian signature, for example, when $\mathcal{M}$ is the Kerr-NUT-(A)dS metric or the Taub-NUT metric [58-63]. Second, it will not be possible to lift a generic CKY tensor on $\mathcal{M}$ to a CKY tensor on $\hat{\mathcal{M}}$. This will be discussed in more detail in Sec. IV, where it will be shown that the process of lift/oxidation and its inverse, reduction, at the level of the Dirac equation involves a nontrivial projection in phase space, and this is not compatible with all lower and higher dimensional hidden symmetries.

\section{Ansatz 1}

Consider the $p$ form $\hat{f}_{1}$ on $\hat{\mathcal{M}}$ given by (3.8). Let us check under which conditions this satisfies the $(n+2)$ dimensional CKY equation (3.7).

The CKY equation (3.7) splits into three types of equation, one for each of $\hat{X}=\hat{X}^{+}, \hat{X}=\hat{X}^{-}$, and $\hat{X}=\hat{X}^{a}$. We can analyze each using Eqs. (C2)-(C6). The $\hat{X}=\hat{X}^{+}$ component gives

$$
\partial_{v} f=0 \text {, }
$$




$$
\delta f=0,
$$

the $\hat{X}=\hat{X}^{-}$component gives

$$
\begin{array}{r}
\left.d V^{\sharp}\right\lrcorner f=0, \\
F \underset{2}{\wedge} f=0, \\
\partial_{t} f+\frac{p+1}{p} \frac{e}{2 m} F \wedge f=0,
\end{array}
$$

and, last, the $\hat{X}=\hat{X}^{a}$ component gives

$$
\begin{array}{r}
\left.\left.\frac{e}{2 m}\left(X_{a}\right\lrcorner F\right) \wedge \underset{1}{\wedge} f=\frac{1}{p+1} X_{a}\right\lrcorner \partial_{t} f, \\
\left.\nabla_{a} f=\frac{1}{p+1} X_{a}\right\lrcorner d f .
\end{array}
$$

The latter equation is the Killing-Yano equation on the base. The former instead implies (3.16).

Thus we have a $t$-parametrized family of KY forms on $\mathcal{M}$. But this is compatible with Eq. (3.16) only if $F \wedge_{1} f$ is $\mathrm{KY}$ as well, which in general will not be the case. Then it must be that separately

$$
\begin{gathered}
\partial_{t} f=0, \\
\left.\left(X_{a}\right\lrcorner F\right) \wedge f
\end{gathered}
$$

which implies

$$
F \wedge f=0 .
$$

Thus there is no $v, t$ dependency, and we discover that a KY form on the base manifold $\mathcal{M}$ can be lifted directly to a $\mathrm{KY}$ form on $\hat{\mathcal{M}}$, since the conditions found imply $\hat{\delta} \hat{f}_{1}=0$. With such a form we can construct a symmetry operator for the Dirac equation on $\hat{\mathcal{M}}$, and when $p$ is odd we know that such an operator strictly commutes with the Dirac operator $\hat{D}$ [41]. Also for such values of $p$ the conditions we have found in this section guarantee that on $\mathcal{M}$ we can build a symmetry operator for the Dirac equation with $V$ and $A$ flux [38]. In Sec. IV we show how in the case of $p$ odd it is possible to dimensionally reduce such a hidden symmetry operator on $\hat{\mathcal{M}}$ to get a hidden symmetry operator associated with flux on $M$. We will also see how this is not possible if $p$ is even, which goes in agreement with the fact that the conditions required on an even CKY tensor with flux in Ref. [38] are different. Such other conditions are those to be found in Sec. III B 4. It is worthwhile to realize that the conditions found here are more restrictive than those in Ref. [38], so in general it is possible to conceive the existence of tensors that satisfy the less restrictive conditions, thus generating symmetries of the Dirac equation with flux on $\mathcal{M}$, but that at the same time do not satisfy the conditions of this section and therefore cannot be lifted to $\hat{\mathcal{M}}$.

Last, we notice that since Hodge duality exchanges KY with CCKY forms, the result of this section implies that the form $\hat{f}_{4}$ of Eq. (3.11) is expected to be a CCKY form on $\hat{\mathcal{M}}$, with the $f$ function appearing there a CCKY form on $\mathcal{M}$.

\section{Ansatz 2}

Consider the $(p+1)$ form $\hat{f}_{2}$ on $\hat{\mathcal{M}}$ given by

$$
\hat{f}_{2}=\hat{e}^{+} \wedge f \text {. }
$$

We can calculate derivatives of $\hat{f}_{2}$ and get

$$
\begin{gathered}
\hat{\nabla}_{-} \hat{f}_{2}=\hat{e}^{+} \wedge \partial_{v} f, \\
\hat{\nabla}_{+} \hat{f}_{2}=\hat{e}^{+} \wedge\left[\left(\frac{V}{m} \partial_{v}+\partial_{t}\right) f+\frac{e}{2 m}(F \wedge f)\right], \\
\hat{\nabla}_{a} \hat{f}_{2}=\hat{e}^{+} \wedge\left(-\frac{e}{m} A_{a} \partial_{v} f+\nabla_{a} f\right), \\
\hat{d} \hat{f}_{2}=-\hat{e}^{+} \wedge \hat{e}^{-} \wedge \partial_{v} f+\hat{e}^{+} \wedge\left(\frac{e}{m} A \wedge \partial_{v} f-d f\right), \\
\left.\hat{\delta} \hat{f}_{2}=-\partial_{v} f+\hat{e}^{+} \wedge\left(-\frac{e}{m} A^{\sharp}\right\lrcorner \partial_{v} f-\delta f\right) .
\end{gathered}
$$

The $\hat{X}=\hat{X}^{+}$component of the CKY equation gives

$$
\partial_{v} \hat{f}=0,
$$

the $\hat{X}=\hat{X}^{-}$component gives

$$
\begin{aligned}
\delta \hat{f} & =0, \\
d \hat{f} & =0, \\
\partial_{t} \hat{f}+\frac{e}{2 m} F \underset{1}{\hat{f}} & =0,
\end{aligned}
$$

and, last, the $\hat{X}=\hat{X}^{a}$ component gives

$$
\nabla_{a} \hat{f}=0 .
$$

So in this case $f$ and $\hat{f}_{2}$ are covariantly constant forms.

\section{Ansatz 3}

Consider the $(p+1)$ form $\hat{f}$ on $\hat{\mathcal{M}}$ given by

$$
\hat{f}_{3}=\hat{e}^{-} \wedge f \text {. }
$$

The explicit form of the derivatives of $\hat{f}_{3}$ is

$$
\hat{\nabla}_{-} \hat{f}_{3}=\hat{e}^{-} \wedge \partial_{v} f
$$




$$
\begin{aligned}
\hat{\nabla}_{+} \hat{f}_{3}= & \hat{e}^{-} \wedge \\
+ & \left.\hat{e}^{+} \wedge \hat{e}^{-} \wedge\left(\frac{d V^{\sharp}}{m}\right\lrcorner f\right)+\frac{d V}{m} \wedge f \\
\hat{\nabla}_{a} \hat{f}_{3}= & \hat{e}^{-} \wedge\left(-\frac{e}{m} A_{a} \partial_{v} f+\nabla_{a} f\right) \\
+ & \left.\left.\frac{e}{2 m} \hat{e}^{+} \wedge \hat{e}^{-} \wedge\left[\left(X_{a}\right\lrcorner F\right) \wedge f\right]+\frac{e}{2 m}\left(X_{a}\right\lrcorner F\right) \wedge f,
\end{aligned}
$$

$$
\begin{aligned}
\hat{d} \hat{f}_{3}= & \hat{e}^{+} \wedge \frac{d V}{m} \wedge f+\hat{e}^{-} \wedge\left(\frac{e}{m} A \wedge \partial_{v} f-d f\right) \\
& +\hat{e}^{+} \wedge \hat{e}^{-} \wedge\left(\frac{V}{m} \partial_{v}+\partial_{t}\right) \hat{f}+\frac{e}{m} F \wedge f, \\
\hat{\delta} \hat{f}_{3}= & \left.\left.\hat{e}^{+} \wedge\left(\frac{d V^{\#}}{m}\right\lrcorner f\right)+\hat{e}^{-} \wedge\left(-\frac{e}{m} A^{\#}\right\lrcorner \partial_{v} f-\delta f\right) \\
& +\hat{e}^{+} \wedge \hat{e}^{-} \wedge\left(\frac{e}{2 m} F \wedge f\right) \\
& -\left[\left(\frac{V}{m} \partial_{v}+\partial_{t}\right) \hat{f}+\frac{e}{2 m} F \wedge f\right] .
\end{aligned}
$$

The CKY equation gives again a covariantly constant case, with $\partial_{v} f=0=\partial_{t} f, \nabla_{a} f=0$.

\section{Ansatz 4}

Last, consider the $(p+2)$ form $\hat{f}_{4}$ on $\hat{\mathcal{M}}$ given by

$$
\hat{f}_{4}=\hat{e}^{+} \wedge \hat{e}^{-} \wedge f
$$

Its derivatives are given by

$$
\begin{gathered}
\hat{\nabla}_{-} \hat{f}_{4}=\hat{e}^{+} \wedge \hat{e}^{-} \wedge \partial_{v} f \\
\hat{\nabla}_{+} \hat{f}_{4}=\hat{e}^{+} \wedge \frac{1}{m} d V \wedge f+\hat{e}^{+} \wedge \hat{e}^{-} \wedge\left[\left(\frac{V}{m} \partial_{v}+\partial_{t}\right) f\right. \\
\left.+\frac{e}{2 m} F \wedge f\right] \\
\left.\hat{\nabla}_{a} \hat{f}_{4}=\hat{e}^{+} \wedge \frac{e}{2 m}\left(X_{a}\right\lrcorner F\right) \wedge f \\
+\hat{e}^{+} \wedge \hat{e}^{-} \wedge\left[-\frac{e}{m} A_{a} \partial_{v} f+\nabla_{a} f\right] \\
\hat{d} \hat{f}_{4}=-\hat{e}^{+} \wedge \frac{e}{m} F \wedge f+\hat{e}^{+} \wedge \hat{e}^{-} \wedge\left(-\frac{e}{m} A \wedge \partial_{v} f+d f\right),
\end{gathered}
$$

$$
\begin{aligned}
\hat{\delta} \hat{f}_{4}= & -\hat{e}^{-} \wedge \partial_{v} f+\hat{e}^{+} \wedge\left(\frac{V}{m} \partial_{v}+\partial_{t}\right) f \\
& \left.+\hat{e}^{+} \wedge \hat{e}^{-} \wedge\left(\frac{e}{m} A^{\#}\right\lrcorner \partial_{v} f+\delta f\right) .
\end{aligned}
$$

These are exactly the Hodge dual of the equations for $\hat{d} \hat{f}_{1}$ and $\hat{\delta} f_{1}$ found in Sec. III B 1 , with the understanding that the form $f$ there is related to the $f$ form of this section by Hodge duality on the base manifold $\mathcal{M}$. The CKY equations therefore lead to the Hodge dual of the conditions found there, as it can be checked using the identities in Appendix B, namely,

$$
\begin{gathered}
\partial_{v, t} f=0, \\
d f=0, \\
d V \wedge f=0, \\
\left.\left(X_{a}\right\lrcorner F\right) \wedge g=0, \\
F \wedge f=0, \\
F \wedge f=0, \\
\nabla_{a} f=-\frac{1}{n-p+1} e_{a} \wedge \delta f .
\end{gathered}
$$

There are two things worth noticing. The first is that Eq. (3.51) is the CCKY equation on the base manifold and that this is ultimately made possible by the fact that $n-p+1=(n+2)-(p+2)+1$. When all the conditions are satisfied, $\hat{f}_{4}$ is a CCKY tensor on $\hat{\mathcal{M}}$. The second thing worth noticing is that the remaining conditions guarantee that if $p$ is even, we can build a symmetry operator of the Dirac equation on $\hat{\mathcal{M}}$ and of the Dirac equation with $V$ and $A$ flux on $\mathcal{M}$ [38]. These conditions complement those found in Sec. III B 1 and are associated with the dimensional reduction to $\mathcal{M}$ of a symmetry operator on $\hat{\mathcal{M}}$, as will be discussed in more detail in Sec. IV. In this case, too, the conditions are stronger than those found in Ref. [38].

\section{The general CKY tensor}

We are now ready to tackle the general case. Let the $p$ form $\hat{f}$ be parametrized as

$$
\hat{f}=f+\hat{e}^{+} \wedge \rho^{+}+\hat{e}^{-} \wedge \rho^{-}+\hat{e}^{+} \wedge \hat{e}^{-} \wedge g,
$$

where the forms $f, \rho^{ \pm}$, and $g$ are $p, p-1$, and $p-2$ forms, respectively, defined on $\mathcal{M}$. We can calculate all its derivatives by adding the derivatives calculated in the four previous ansätze. The full conditions obtained from the CKY equation are somewhat long and are listed in Appendix D. We are interested here in discussing the special case where the forms $f, \rho^{ \pm}$, and $g$ do not depend explicitly on the $v, t$ coordinates, or, in other words, the Lie derivative of $\hat{f}$ with respect to the Killing vectors $\partial_{v}$ and $\partial_{t}$ 
is zero. In this case we can relate the parametrization (3.52) to the lift of a set of forms defined on the base manifold $\mathcal{M}$, while in the most general case listed in the Appendix D it is necessary to consider $v$ and $t$ parametrized families of forms.

In general, the CKY equation reduces to a series of equations on $\mathcal{M}$. We label these according to two indices $i$ and $J$ according to the following convention. The first index $i=-,+, a$ reflects which derivative is used in the CKY equation among $\hat{\nabla}_{-}, \hat{\nabla}_{+}$, and $\hat{\nabla}_{a}$. Once the appropriate CKY equation is chosen, the second index $J$, taking values $J=+,-$, \pm , $a$, is related to taking the projection on $\mathcal{M}$ of the CKY equation along the $\hat{e}^{+}, \hat{e}^{-}, \hat{e}^{+} \wedge \hat{e}^{-}$, and $\hat{e}^{a}$ direction. The full set of equations is

$$
\begin{aligned}
& \left.(-+) d g=0, \quad(-+)^{\prime} \delta f=0 ; \quad(+-) F \wedge \rho_{1}^{-}=0, \quad(++)-\frac{d V^{\#}}{m}\right\lrcorner f+\frac{d V}{m} \wedge g+\frac{e}{2 m} F \wedge \rho^{+}=0, \\
& \left.(+ \pm) \frac{e}{2 m} F \wedge g=+\frac{1}{n+3-p}\left[\frac{e}{2 m} F \wedge f-\delta \rho^{+}-(n+2-p) \frac{d V^{\sharp}}{m}\right\lrcorner \rho^{-}\right]=0, \\
& (+a) \frac{e}{2 m} F \wedge f=+\frac{1}{p+1}\left[-d \rho^{+}-p \frac{d V}{m} \wedge \rho^{-}-\frac{e}{m} F \wedge g\right], \quad(a-) \nabla_{a} \rho^{-}=0, \\
& \left.\left.\left.(a+)-\frac{e}{2 m}\left(X_{a}\right\lrcorner F\right) \wedge f+\nabla_{a} \rho^{+}+\frac{e}{2 m}\left(X_{a}\right\lrcorner F\right) \wedge g=\frac{X_{a}}{p+1}\right\lrcorner\left[d \rho^{+}-\frac{d V}{m} \wedge \rho^{-}+\frac{e}{m} F \wedge g\right] \\
& \left.+\frac{e_{a}}{n+3-p} \wedge\left[\frac{e}{2 m} F \wedge f-\delta \rho^{+}+\frac{d V^{\sharp}}{m}\right\lrcorner \rho^{-}\right], \\
& \left.(a \pm) \nabla_{a} g+\frac{e}{2 m}\left(X_{a}\right\lrcorner F\right) \wedge \rho_{1}^{-}=-\frac{e_{a}}{n+3-p} \wedge\left[\delta g+\frac{e}{2 m} F \wedge \rho^{-}\right], \\
& \left.\left.(a b) \nabla_{a} f+\frac{e}{2 m}\left(X_{a}\right\lrcorner F\right) \wedge \rho^{-}=\frac{X_{a}}{p+1}\right\lrcorner\left[+d f+\frac{e}{m} F \wedge \rho^{-}\right] .
\end{aligned}
$$

These equations classify the most general CKY tensor on $\hat{\mathcal{M}}$ that has zero Lie derivative with respect to $\partial_{v}$ and $\partial_{t}$ in terms of forms on $\mathcal{M}$. The last two equations are, respectively, a CCKY and a KY equation for $g$ and $f$, respectively, with a deformation parametrized by the covariantly constant form $\rho^{-}$and by $F$. The form $\rho^{+}$instead is related to a deformation of the equations with no derivatives for $f$ and $g$ that we have found in the previous sections for terms of the kind $d V \wedge, d V\lrcorner$, and $F \wedge_{1}$.

In particular, it can be seen that under no circumstance can the form $f$ be strictly CCKY with $\delta f \neq 0$ or can the form $g$ be strictly KY with $d g \neq 0$, thus proving that if any such form exists on $\mathcal{M}$, then it cannot be lifted to $\hat{\mathcal{M}}$ in order to give a CKY form. The forms $\rho^{ \pm}, f$, and $g$ satisfy a set of generalized interdependent CKY equations.

\section{Conditions for rank 2 CCKY tensors}

It is of particular interest to discuss rank $2 \mathrm{CCKY}$ tensors in the Eisenhart-Duval lift metrics given the fact there exists a classification of such tensors for Riemannian spacetimes. Therefore an interesting question is whether Eisenhart-Duval spacetimes can provide new nontrivial examples of such forms.

If the form $\hat{f}$ of the previous section is of rank 2, then $g$ is a function and $\rho^{ \pm}$are 1 forms. Equation $(-+): d g=0$ then tells us that $g$ is constant. Asking that $\hat{f}$ is closed amounts to the two conditions

$$
\begin{array}{r}
d f+\frac{e}{m} F \wedge \rho^{-}=0, \\
d \rho^{+}-\frac{d V}{m} \wedge \rho^{-}+\frac{e}{m} g F=0,
\end{array}
$$

which have as solutions

$$
\begin{gathered}
f=-\frac{e}{m} A \wedge \rho^{-}+d \Lambda^{(1)}, \\
\rho^{+}=\frac{V}{m} \rho^{-}-\frac{e g}{m} A+d \Lambda^{(0)},
\end{gathered}
$$

where $\Lambda^{(0)}$ and $\Lambda^{(1)}$ are a 0 and a 1 form, respectively, to be found by solving the other equations.

The full set of equations are complicated to solve. One might hope to obtain a simplification in the case $F=0$, $V \neq 0$. If $F=0$, then equation $(++)$ gives

$$
\left.\frac{d V^{\#}}{m}\right\lrcorner f=g \frac{d V}{m} .
$$

Taking the hook contraction of this equation with $d V^{\sharp}$, we get $0=g \frac{|\vec{\nabla} V|^{2}}{m}$, and since $\mathcal{M}$ has a Euclidean signature, this means that either $g=0$ or $V=$ const. We take $g=0$. The rest of the equations simplify to 
$(-+)^{\prime} \delta f=0$,

$\left.(++) \frac{d V^{\#}}{m}\right\lrcorner f=0$,

$\left.(+ \pm) \delta \rho^{+}=-n \frac{d V^{\sharp}}{m}\right\lrcorner \rho^{-}$,

$(+a) \quad d \rho^{+}=-2 \frac{d V}{m} \wedge \rho^{-}$,

$(a-) \quad \nabla_{a} \rho^{-}=0$,

$\left.(a+) \nabla_{a} \rho^{+}=\frac{e_{a}}{n+1} \wedge\left(-\delta \rho^{+}+\frac{d V^{\sharp}}{m}\right\lrcorner \rho^{-}\right)$,

(ab) $\quad \nabla_{a} f=0$.

Now we can see that equation $(a+)$ implies $d \rho^{+}=0$, and this together with $(+a)$ implies $d V \wedge \rho^{-}=0$, or

$$
\frac{V}{m}=\alpha \rho^{-} \cdot \vec{x}+\beta,
$$

where $\alpha$ and $\beta$ are constants.

Now $(+ \pm)$ together with $(a+)$ give

$$
\left.\nabla_{a} \rho^{+}=\left(\frac{d V^{\sharp}}{m}\right\lrcorner \rho^{-}\right) e_{a}=\alpha\left|\rho^{-}\right|^{2} e_{a},
$$

and this together with Eq. (3.57) yields the following equation for the form $\Lambda^{(0)}$ :

$$
\nabla_{a}\left(d \Lambda^{(0)}\right)=-\alpha\left(\rho_{a}^{-} \rho_{b}^{-}-\left|\rho^{-}\right|^{2} \delta_{a b}\right) e^{b} .
$$

One might try to solve this equation when $\mathcal{M}$ is the Euclidean flat space. A solution can indeed be displayed; however, in this case $\hat{M}$ is flat, and therefore this corresponds to no new metrics. Regardless of the specific form of $\mathcal{M}$ the conditions we found so far guarantee that the codifferential of $\hat{f}$ is a null form:

$$
\hat{\delta} \hat{f}=(n+1) \alpha\left(\rho^{-}\right)^{2} e^{+} .
$$

We do not pursue here the task of solving (3.62) when $\mathcal{M}$ is a nonflat space, or that of solving the full set of equations when $F \neq 0$. However, we consider this an interesting task given its potential to generate new metrics with rank 2 CCKY tensors that are not covered by the Riemannian classification.

\section{DIRAC EQUATION}

In this section we describe in detail the massless Dirac equation on $\hat{\mathcal{M}}$ and show that in general it gives rise to a nonrelativistic Lévy-Leblond equation. We then specialize to those special cases when it is possible to reduce the higher dimensional Dirac equation and obtain again a Dirac equation in lower dimension. First, we show how an appropriate nontrivial projection on the higher dimensional spinor allows one to reduce the massless Dirac equation on $\hat{\mathcal{M}}$ to the Dirac equation with $V$ and $A$ flux on $\mathcal{M}$. We also show how to perform the inverse lift operation, that is, how to embed a lower dimensional solution of the Dirac equation with flux into a solution of the massless higher dimensional Dirac equation. Second, we consider the dimensional reduction of hidden symmetry operators associated with the KY and CCKY tensors of Secs. III B 1 and III B 4, showing how only a subset of these commutes with projection operation and thus yields hidden symmetry operators for the theory on $\mathcal{M}$.

\section{A. Dimensional reduction and lift}

\section{Nonrelativistic Lévy-Leblond equation}

In Ref. [5] it has been shown how the massless free Klein-Gordon equation in $(d+2)$-dimensional Lorentzian spacetime can be reduced to the massive Schrödinger equation in Riemannian $d$-dimensional spacetime using a projection on the base space $\mathcal{M}$ of the Eisenhart-Duval spacetime $\hat{\mathcal{M}}$. In this section we show analogously how dimensional reduction of the massless Dirac equation on $\hat{\mathcal{M}}$ yields its nonrelativistic counterpart on $\mathcal{M}$, that is, the Lévy-Leblond equation [64]. The first derivation of the Lévy-Leblond equation from a lightlike reduction from 4 and 5 dimensions has been given in Ref. [65].

Spinors on $\mathcal{M}$ have dimension $2^{\left[\frac{n}{2}\right]}$, while spinors on $\hat{\mathcal{M}}$ have dimension $2^{2^{\left.\frac{[n+2}{2}\right]}}=2 \cdot 2^{\left[\frac{n}{2}\right]}$. Given the Pauli matrices

$$
\begin{aligned}
\sigma_{1} & \equiv\left(\begin{array}{cc}
0 & 1 \\
1 & 0
\end{array}\right), \\
\sigma_{2} & \equiv\left(\begin{array}{cc}
0 & -i \\
i & 0
\end{array}\right), \\
\sigma_{3} & \equiv\left(\begin{array}{cc}
1 & 0 \\
0 & -1
\end{array}\right),
\end{aligned}
$$

we define $\sigma^{ \pm}=\frac{\sigma_{1} \pm i \sigma_{2}}{2}$. These satisfy $\left(\sigma^{ \pm}\right)^{2}=0$, $\left\{\sigma^{+}, \sigma^{-}\right\}=\rrbracket$. Let $\Gamma^{a}$ be gamma matrices for $\mathcal{M}$, and $\hat{\Gamma}^{A}$ for $\hat{\mathcal{M}}$. We use the following representation for gamma matrices on $\hat{\mathcal{M}}$ :

$$
\hat{\Gamma}^{+}=\sigma^{+} \otimes \mathbb{\rrbracket}, \quad \hat{\Gamma}^{-}=\sigma^{-} \otimes \mathbb{}, \quad \hat{\Gamma}^{a}=\sigma_{3} \otimes \Gamma^{a} .
$$

Using the explicit form of the spin connection (A5), we find the expression for the covariant derivatives of a spinor $\hat{\psi}$ on $\hat{\mathcal{M}}$ :

$$
\begin{aligned}
& \hat{\nabla}_{-}=\partial_{v}, \quad \hat{\nabla}_{+}=\left(\frac{V}{m} \partial_{v}+\partial_{t}\right)-\frac{1}{2 m} \hat{\Gamma}^{+} d V-\frac{e}{4 m} F, \\
& \hat{\nabla}_{a}=\nabla_{a}-\frac{e}{m} A_{a} \partial_{v}+\frac{e}{4 m} \hat{\Gamma}^{+} \hat{\Gamma}^{b} F_{b a} .
\end{aligned}
$$

Thus we can write the Dirac operator on $\hat{\mathcal{M}}$ as 


$$
\begin{aligned}
\hat{D}= & \hat{\Gamma}^{A} \hat{\nabla}_{A} \\
= & \hat{\Gamma}^{-} \partial_{v}+\hat{\Gamma}^{+}\left(\frac{V}{m} \partial_{v}+\partial_{t}+\frac{e}{4 m} F\right) \\
& +\hat{\Gamma}^{a}\left(\nabla_{a}-\frac{e}{m} A_{a} \partial_{v}\right) .
\end{aligned}
$$

In the case of a Killing vector $\hat{K}$ the symmetry operators of Eq. (2.16) assume the form

$$
\hat{S}_{\hat{K}}=\hat{\nabla}_{\hat{K}}+\frac{1}{4} \hat{d} \hat{K}
$$

For the two Killing vectors $\hat{X}^{+}$and $\hat{X}^{-}-\frac{V}{m} \hat{X}^{+}$of the lift metric the operators can be calculated explicitly and are given by

$$
K_{\hat{X}^{+}}=\partial_{v}
$$

and

$$
K_{\left(\hat{X}^{-}-\frac{V}{m} \hat{X}^{+}\right)}=\partial_{t} .
$$

They both commute with the Dirac operator $\hat{D}$ on $\hat{\mathcal{M}}$, so we can ask that a spinor $\hat{\psi}$ on $\hat{\mathcal{M}}$ that satisfies $\hat{D} \hat{\psi}=0$ is also an eigenspinor of the two operators. For the purpose of recovering the Lévy-Leblond equation we ask the less restrictive condition of $\hat{\psi}$ being eigenspinor only of $K_{\hat{X}^{+}}$:

$$
\partial_{v} \hat{\psi}=i m \hat{\psi} .
$$

We have chosen the $\partial_{v}$ eigenvalue to be proportional to the mass parameter $m$ in order for the Dirac operator to reduce to

$$
\hat{D}=i m \hat{\Gamma}^{-}+\hat{\Gamma}^{+}\left[i V+\partial_{t}+\frac{e}{4 m} F\right]+\hat{\Gamma}^{a} \mathcal{D}_{a},
$$

where $\mathcal{D}_{a}=\nabla_{a}-i e A_{a}$ is the $U(1)$ covariant spinor derivative on $\mathcal{M}$. We also define the Dirac operator on $\mathcal{M}$ with $A$ flux as $\mathcal{D}=\Gamma^{a} \mathcal{D}_{a}$.

According to the gamma matrices representation (4.2), we write a spinor $\hat{\psi}$ on $\hat{\mathcal{M}}$ as

$$
\hat{\psi}=\left(\begin{array}{l}
\chi_{1} \\
\chi_{2}
\end{array}\right)
$$

where $\chi_{1}$ and $\chi_{2}$ are spinors on $\mathcal{M}$. Then the massless Dirac equation on $\hat{\mathcal{M}}, \hat{D} \hat{\psi}=0$, can be written as

$$
\left(\begin{array}{cc}
\mathcal{D} & \mathcal{O}+\partial_{t} \\
i m & -\mathcal{D}
\end{array}\right)\left(\begin{array}{l}
\chi_{1} \\
\chi_{2}
\end{array}\right)=0
$$

where the operator $\mathcal{O}$ is given by $\mathcal{O}=i V+\frac{e}{4 m} F$, and it reduces to two equations on $\mathcal{M}$ :

$$
\partial_{t} \chi_{2}+\mathcal{O} \chi_{2}+\mathcal{D} \chi_{1}=0, \quad i m \chi_{1}-\mathcal{D} \chi_{2}=0 .
$$

This is the nonrelativistic Lévy-Leblond equation for a particle of mass $\tilde{m}=\frac{m}{2}$, in curved space, with scalar and vector potential and with an additional term $\frac{e}{4 m} F$. This extra term induces an anomalous gyromagnetic factor $g=$ $3 / 2$ and was not present in the original work by LévyLeblond. To see the value of the gyromagnetic factor, one can in the second equation in Eq. (4.12) find $\chi_{1}$ as a function of $\chi_{2}$ and substitute it back into the first equation, thus getting

$$
\left[E-\frac{1}{2 \tilde{m}}\left(\Pi^{\mu}-e A^{\mu}\right)^{2}-V-i \frac{3 e}{8 \tilde{m}} F-\frac{R}{8 \tilde{m}}\right] \chi_{2}=0,
$$

where $E=i \partial_{t}, \Pi_{\mu}=-i \nabla_{\mu}$ is the momentum, and $R$ is the scalar curvature of $\mathcal{M}$.

In Ref. [65] the anomalous gyromagnetic factor term does not appear; however, there the setting is different: dimensional reduction is done starting from a massless Dirac equation with vector flux on $\hat{M}$, while in the present case the vector potential originates from the metric and not directly from the massless Dirac equation. Therefore the massless Dirac equation considered in Ref. [65] that is dimensionally reduced is not the same as the one considered here. Situations where a dimensional reduction induces an anomalous gyromagnetic factor are not unknown; see Ref. [66] for an example and further references.

This dimensional reduction gives a geometrical derivation of the Lévy-Leblond equation. One of the reasons why this is ultimately possible is the fact that the Bargmann group-the central extension of the Galilei group that leaves invariant the Schrödinger equation and the LévyLeblond equation - can be embedded in the de Sitter group $O(1, n+1)[2]$.

\section{Relativistic Dirac equation: Lift and reduction}

The $\partial_{t}$ term, as seen in the previous section, is of main importance in order to obtain nonrelativistic equations. In this section we seek to understand those cases when the massless Dirac equation on $\hat{\mathcal{M}}$ can be dimensionally reduced to the, still relativistic, Dirac equation with flux on $\mathcal{M}$. As we will see, this cannot always be done differently from the nonrelativistic case, the details of the reduction depending on the explicit form on the scalar and vector potentials. However, at least in the case of $V=m$ and $F=0$ the dimensional reduction and its inverse, the lift, can always be performed.

We start then by asking the following two conditions for $\hat{\psi}$ :

$$
\begin{aligned}
& \partial_{v} \hat{\psi}=i m \hat{\psi}, \\
& \partial_{t} \hat{\psi}=0,
\end{aligned}
$$

which, as seen in the previous section, are compatible with the Dirac equation on $\hat{\mathcal{M}}$. 
The Dirac operator reduces to

$$
\hat{D}=i m \hat{\Gamma}^{-}+\hat{\Gamma}^{+}\left[i V+\frac{e}{4 m} F\right]+\hat{\Gamma}^{a} \mathcal{D}_{a} .
$$

Then the massless Dirac equation on $\hat{\mathcal{M}}, \hat{D} \hat{\psi}=0$, can be written as

$$
\left(\begin{array}{cc}
\mathcal{D} & \mathcal{O} \\
\text { im } & -\mathcal{D}
\end{array}\right)\left(\begin{array}{l}
\chi_{1} \\
\chi_{2}
\end{array}\right)=0
$$

where the operator $\mathcal{O}$ is given by $\mathcal{O}=i V+\frac{e}{4 m} F$. The general solution of $\hat{D} \hat{\psi}=0$ is given by

$$
\hat{\psi}=\left(\begin{array}{c}
-\frac{i}{m} \mathcal{D}_{\chi_{2}} \\
\chi_{2}
\end{array}\right)
$$

where $\chi_{2}$ satisfies

$$
\frac{i}{m} \mathcal{D}^{2} \chi_{2}=\mathcal{O}_{\chi_{2}}
$$

which is an integrability condition of (4.17).

To make contact with the Dirac equation with flux on $\mathcal{M}$, we impose the following condition on the generic spinor $\hat{\psi}$ of (4.10):

$$
i V \chi_{1}=\mathcal{O}_{\chi_{2}}
$$

This allows one to solve for $\chi_{1}$ if $\chi_{2}$ is known and vice versa, since $\mathcal{O}$ is invertible for generic values of $F, V$, and $E$. The reason to ask for this condition is that, when it is satisfied, then the spinor $\chi_{1}$ satisfies

$$
(\mathcal{D}+i V) \chi_{1}=0,
$$

which is the Dirac equation with $V$ and $A$ flux on the base. It is worth noticing that the condition (4.20) is nontrivial and will be satisfied only for specific combinations of $V$ and $F{ }^{1}$

To gain insight into the condition, we can rewrite Eq. (4.20) as a projection $P \hat{\psi}=\hat{\psi}$, where $P$ is the projector

\footnotetext{
${ }^{1} \mathrm{We}$ are not trying to prove here that the projection (4.20) is the only way to obtain a Dirac equation on $\mathcal{M}$. Other methods can be conceived. As a concrete example, when $\hat{\mathcal{M}}$ is a onedimensional Lorentzian manifold, one could consider a Majorana-Weyl spinor, which has 16 real components, and compare it with either a Weyl or a Majorana spinor on $\mathcal{M}$, which is an eight-dimensional Riemannian manifold, which also has 16 real components. It may be hoped that in this way a projection such as (4.20) may not be needed. A concrete calculation, however, shows that the ten-dimensional spinor reduces into a pair of separate eight-dimensional Majorana-Weyl spinors, each of which has eight real components. The ten-dimensional Dirac equation can induce an eight-dimensional massless Dirac equation for one of the spinors on $\mathcal{M}$; however, this is not compatible with (4.14). One has either to set the right-hand side of (4.14) to zero or to set both the other spinor and the fluxes to zero, which amounts to a special choice of Majorana-Weyl spinor on $\hat{\mathcal{M}}$ and of metric. Although this is an interesting possibility, we have not considered it further since we have been focusing on the case where (4.14) holds.
}

$$
P=\frac{\square-i \frac{\mathcal{O}}{V} \hat{\Gamma}^{+}+i \frac{V}{\mathcal{O}} \hat{\Gamma}^{-}}{2} \text {. }
$$

$P$ is well defined when $-i V^{-1} \mathcal{O}=\rrbracket-i \frac{e}{4 m} V^{-1} F$ is invertible, in which case it satisfies $P^{2}=P$.

The following three alternative cases can occur. First, if both $V=0$ and $F=0$, then Eq. (4.20) is always satisfied and $\chi_{1}$ satisfies the massless Dirac equation on the base with no flux, $D \chi_{1}=0$. Second, if instead $V \neq 0$ but $F=0$, then $-i V^{-1} \mathcal{O}=\rrbracket$ and $P=\frac{1}{2}\left(\llbracket+\Gamma^{+}+\Gamma^{-}\right)$. Then $P \hat{\psi}=\hat{\psi}$ has the symmetric solution $\chi_{1}=\chi_{2}$. Third, if both $V \neq 0$ and $F \neq 0$, then for generic values of $V$ and $F$ the operator $P$ will be well defined and the solution of $P \hat{\psi}=\hat{\psi}$ will be a twist of the symmetric case parametrized by $F$.

In the symmetric case $V \neq 0$ and $F=0$, the projector $P$ satisfies the equation

$$
\hat{D} P=-\frac{\Gamma^{+}+\Gamma^{-}}{2} \hat{D}+i(m+V),
$$

which means that for generic values of $V$ it will not be possible to ask that for any spinor $\hat{\psi}$ that satisfies the massless Dirac equation on $\hat{\mathcal{M}}$ then its projection $P \hat{\psi}$ will also satisfy the equation. The only exception to this is for $V=-m$, which corresponds to considering the Dirac equation on $\mathcal{M}$ for a particle of mass $m$. In this case if $\hat{D} \hat{\psi}=0$, then $\hat{D} P \hat{\psi}=0$ and from $P \hat{\psi}$ we can construct a solution of the Dirac equation with mass on $\mathcal{M}$. Considering all possible such $\hat{\psi}$, we can construct all the independent solutions on $\mathcal{M}$. Vice versa, given a solution of the Dirac equation with mass on $\mathcal{M}$, this can be lifted to a solution of the massless Dirac equation on $\hat{\mathcal{M}}$ satisfying $P \hat{\psi}=\hat{\psi}$. On the other hand, a direct substitution of the condition $\chi_{1}=\chi_{2}$ into the Dirac equation (4.17) shows that the case $V=-m$ is the only one consistent with the spinor equations. It is interesting to notice that solutions of the equation $P \hat{\psi}=0$, which are orthogonal to the previous ones, satisfy the condition $-i V \chi_{1}=\mathcal{O} \chi_{2}$. Then these are associated with solutions of the Dirac equation on the base with $-V$ flux.

In the generic case $V \neq 0$ and $F \neq 0$ Eq. (4.23) will also receive contributions proportional to $V^{-1} F$ and its derivatives. This opens the possibility of nontrivial solutions, although these are not easy to analyze in the general case. One possibility is to analyze the compatibility condition between the projection and the Dirac equation. When both hold, then $\chi_{2}$ also has to satisfy the nontrivial equation

$$
\frac{V}{m} \mathcal{D} \chi_{2}=\mathcal{O}_{\chi_{2}}
$$

In Sec. V we show a simple example with nonzero $V$ and $F$ where the projection is compatible with the Dirac equation.

We conclude the discussion showing how to lift a solution of the Dirac equation with flux to a solution of the 
massless Dirac equation on $\hat{\mathcal{M}}$. Suppose there exists a given spinor $\chi_{1}$ on $\mathcal{M}$ satisfying the Dirac equation with flux

$$
\mathcal{D} \chi_{1}+i V \chi_{1}=0
$$

To upgrade $\chi_{1}$ to a spinor on $\hat{\mathcal{M}}$ we can define a spinor $\chi_{2}$ on $\mathcal{M}$ solving

$$
\mathcal{D} \chi_{2}=i m \chi_{1} \text {. }
$$

Now, in order for the spinor $\hat{\psi}=\left(\chi_{1}, \chi_{2}\right)$ to satisfy the massless Dirac equation on $\hat{\mathcal{M}}$, we need to ask that also condition (4.20) holds. Again the number of solutions will not be maximal owing to compatibility conditions.

\section{B. Hidden symmetry operators}

In this section we examine hidden symmetry operators of the two theories from the two complementary points of view of dimensional reduction and lift. From the former, it is to be expected that not all hidden symmetries of the dynamics on $\hat{\mathcal{M}}$ can be reduced to symmetries of the dynamics on $\mathcal{M}$ as the embedding of the lower dimensional theory in the higher dimensional one is a proper inclusion in terms of dynamics. For example, there are KY and CCKY forms on $\hat{\mathcal{M}}$ that arise as lifts of KY and CCKY forms on $\mathcal{M}$ and yet such that their corresponding symmetry operators cannot be dimensionally reduced. This is consistent with the observation made in Secs. III B 1 and III B 4 that according to whether the form is even or odd different conditions are required in order for it to generate a hidden symmetry operator for the Dirac equation with flux on $\mathcal{M}$. Those symmetry operators that cannot be dimensionally reduced are built from even KY or odd CCKY tensors and are those that in lower dimension would generate anomalous symmetry operators. From the point of view of the lift of hidden symmetry operators instead we have seen in Sec. III B that it is possible to have symmetry operators on $\mathcal{M}$ generated by CKY tensors that cannot be lifted to $\hat{\mathcal{M}}$.

At the end of the section we will discuss a simple example in which a CKY tensor on $\mathcal{M}$ that is neither strictly KY nor CCKY, when it exists, gives rise to a symmetry operator of the Dirac equation on $\mathcal{M}$, but this symmetry cannot be lifted to a symmetry operator of the Dirac equation on $\hat{\mathcal{M}}$. While this is not a proof that such a lift is not possible, we hope that such an example can help create an intuition on the underlying reason why the lift cannot be done. A proof follows from the results of Sec. III where it is shown no CKY tensor on $\hat{\mathcal{M}}$ can be written in terms of a CKY form on $\mathcal{M}$ that is neither KY or CCKY.

We start analyzing dimensional reduction. We consider the $\mathrm{KY}$ form $\hat{f}_{1}$ on $\hat{\mathcal{M}}$ that is obtained by lifting a $\mathrm{KY} p$ form $f$ on $\mathcal{M}$, as in Sec. III B 1 , and its associated symmetry operator of the Dirac equation given by Eq. (2.16).
From the results of that section, and using Eqs. (C5) and (C6), one can see that $\hat{\delta} \hat{f}_{1}=0$, and $\hat{d} \hat{f}_{1}=d f$. Then in terms of explicit components we can write

$$
\begin{aligned}
\hat{S}_{\hat{f}_{1}}= & \frac{1}{(p-1) !}\left[\hat{\Gamma}^{B_{1} \ldots B_{p-1}} \hat{f}_{1 B_{1} \ldots B_{p-1}}^{A} \hat{\nabla}_{A}\right. \\
& \left.+\frac{1}{2(p+1)^{2}} \hat{\Gamma}^{A_{1} \ldots A_{p+1}} \hat{d} \hat{f}_{1 A_{1} \ldots A_{p+1}}\right] \\
= & \frac{\left(\sigma_{3}\right)^{p-1}}{(p-1) !} \otimes\left[\Gamma^{b_{1} \ldots b_{p-1}} f_{b_{1} \ldots b_{p-1}}^{a}\left(\nabla_{a}-\frac{e}{m} A_{a} \partial_{v}\right)\right. \\
& \left.+\frac{1}{2(p+1)^{2}} \Gamma^{a_{1} \ldots a_{p+1}} d f_{a_{1} \ldots a_{p+1}}\right] .
\end{aligned}
$$

Notice that there is a term proportional to $\left.\left.\left(X^{a}\right\lrcorner f\right) \hat{\Gamma}^{+}\left(X_{a}\right\lrcorner F\right)$ coming from $\hat{\nabla}$ that drops out owing to Eqs. (3.15) and (3.21) and the identities (2.8) and (2.9). The operator above is a symmetry operator of the Dirac operator on $\hat{\mathcal{M}}$; in other words, it transforms solutions of the equation in other solutions. However, to see whether it can be dimensionally reduced to a symmetry operator of the Dirac equation with flux on $\mathcal{M}$, we need to check whether its action commutes with the projection (4.20). If it does, then its action generates an action on the space of solutions of the Dirac equation with flux into itself.

First of all, we notice that when the higher dimensional spinor $\hat{\psi}$ satisfies condition (4.14) then the action of $\hat{S}_{\hat{f}_{1}}$ is the same as that of

$$
\left(\sigma_{3}\right)^{p-1} \otimes \mathcal{S}_{f},
$$

where

$$
\begin{aligned}
\mathcal{S}_{f}= & \frac{1}{(p-1) !} \otimes\left[\Gamma^{b_{1} \ldots b_{p-1}} f_{b_{1} \ldots b_{p-1}}^{a} \mathcal{D}_{a}\right. \\
& \left.+\frac{1}{2(p+1)^{2}} \Gamma^{a_{1} \ldots a_{p+1}} d f_{a_{1} \ldots a_{p+1}}\right]
\end{aligned}
$$

is an operator on $\mathcal{M}$ that formally is the same as a symmetry operator generated from $f$ but such that it uses the $U(1)$ covariant derivative instead of the spinor derivative. Let us then define a new solution $\hat{\psi}^{\prime}$ by

$\hat{\psi}^{\prime}=\left(\begin{array}{l}\chi_{1}^{\prime} \\ \chi_{2}^{\prime}\end{array}\right)=\left(\sigma_{3}\right)^{p-1} \otimes \mathcal{S}_{f}\left(\begin{array}{l}\chi_{1} \\ \chi_{2}\end{array}\right)=\left(\begin{array}{c}\mathcal{S}_{f} \chi_{1} \\ (-1)^{p-1} \mathcal{S}_{f} \chi_{2}\end{array}\right)$,

and check whether $\hat{\psi}^{\prime}$ satisfies (4.20). First of all, notice that $\left.\left.\left[i V, \mathcal{S}_{f}\right] \propto\left(X^{a}\right\lrcorner f\right) \partial_{a} V=d V\right\lrcorner f=0$. Then $i V \chi_{1}^{\prime}=$ $\mathcal{S}_{f} i V \chi_{1}$. Second, since $\hat{\psi}$ satisfies the Dirac equation, then

$$
\begin{aligned}
\mathcal{O}_{\chi_{2}^{\prime}} & =\frac{i}{m} \mathcal{D}^{2} \chi_{2}^{\prime}=\frac{i}{m} \mathcal{D}^{2}\left((-1)^{p-1} \mathcal{S}_{f} \chi_{2}\right) \\
& =(-1)^{p-1} \frac{i}{m} \mathcal{S}_{f} \mathcal{D}^{2} \chi_{2}=(-1)^{p-1} \mathcal{S}_{f} \mathcal{O} \chi_{2}
\end{aligned}
$$

In the last equality we have used the result found in Ref. [38] that $\mathcal{S}_{f}$ graded commutes with $\mathcal{D}$, and therefore commutes with $\mathcal{D}^{2}$. Then 


$$
i V \chi_{1}^{\prime}=\mathcal{S}_{f} i V \chi_{1}=\mathcal{S}_{f} \mathcal{O} \chi_{2}=(-1)^{p-1} \mathcal{S}_{f} \mathcal{O} \chi_{2}^{\prime} .
$$

This proves that the symmetry operator $\hat{S}_{\hat{f}_{1}}$ commutes with the projection (4.20) if and only if $p$ is odd. Then in this case it generates a symmetry operator of the Dirac equation with flux on $\mathcal{M}$, by looking at its action on the spinor $\chi_{1}$. Such an action is given in terms of the operator $\mathcal{S}_{f}$, which is the symmetry operator found in Ref. [38]. In this reference it was found that a subset of the conditions found in Sec. III B 1, namely, Eqs. (3.14), (3.18), and (3.21), guarantee that $\mathcal{S}_{f}$ is a symmetry operator of the Dirac equation with flux, and the results of this section provide a geometrical alternative proof of the original result. ${ }^{2}$

A similar analysis can be done with respect to the CCKY tensor $\hat{f}_{4}$ discussed in Sec. III B 4. We consider the symmetry operator (2.18). This can be written down explicitly and dimensionally reduced when $p$ is even. Now the conditions found in Sec. III B 4 are required to see that the $F$ term present in the spinor derivative $\hat{\nabla}$ drops out and that the action of the operator commutes with that of the projection (4.20). Again, the conditions found are a subset of those found in Ref. [38] for the case of even tensors.

With this we have shown concretely how not all the higher dimensional symmetry operators corresponding to CKY tensors can be dimensionally reduced to give symmetry operators in lower dimension. In particular, this happens also for a subset of the tensors found in Secs. III B 1 and III B 4, which were obtained as a lift of CKY tensors on $\mathcal{M}$. Those lifted tensors such that their higher dimensional symmetry operators do not commute with the projection (4.20) are exactly those for which in lower dimension the corresponding symmetry operators are anomalous owing to the presence of flux. This provides a geometrical interpretation of the anomaly. It should be noticed that the hidden symmetries compatible with the lift, given by odd KY and even CCKY tensors, are exactly those that give rise to operators that fully commute with the Dirac operator [41].

Now we turn to the last objective of this section, providing an intuitive explanation why not all of the symmetry operators found in lower dimension can be lifted. For the calculations to be simpler, suppose there is no flux: $V \equiv 0$, $F \equiv 0$. Let us assume that on $\mathcal{M}$ there exists a CKY $p$ form $\omega$ that is neither KY nor CCKY. Then from Eq. (2.17) we know that

$$
D S_{\omega}=(-1)^{p-1} S_{\omega} D-\frac{(-1)^{p-1}}{n-p+1} \delta \omega D .
$$

Suppose we try to lift $S_{\omega}$ to an operator on $\hat{\mathcal{M}}$ of the kind $\hat{S}=\left(\sigma_{3}\right)^{p-1} \otimes S_{w}$. This is not the only possible way of

\footnotetext{
${ }^{2}$ The conditions found in Ref. [38] are more general than those found here, the most general possible. Correspondingly, not all of the generalized CKY tensors found there can be lifted in the present context to CKY tensors on $\hat{\mathcal{M}}$, as discussed in Sec. III.
}

doing the lift, and therefore this is not a proof, but rather an illustration. Then

$$
\begin{aligned}
\hat{D} \hat{S} & =\left(\hat{\Gamma}^{-} \partial_{v}+\hat{\Gamma}^{+} \partial_{t}+\sigma_{3} \otimes D\right)\left(\sigma_{3}\right)^{p-1} \otimes S_{w} \\
& =(-1)^{p-1} \hat{S} \hat{D}+\frac{(-1)^{p}}{n-p+1}\left(\sigma_{3}\right)^{p} \delta \omega D .
\end{aligned}
$$

If $\hat{\psi}$ is a generic solution of the higher dimension Dirac equation, the first term is zero but in general the second will not be since $\delta \omega \neq 0$ and $D \chi_{2} \neq 0$. This corresponds to the fact that there is no CKY tensor on $\hat{\mathcal{M}}$ that can be written in terms of such a form $\omega$ on $\mathcal{M}$. So the impossibility to perform the lift seems related to the doubling of the spinor degrees of freedom in higher dimension and the fact that the higher dimensional Dirac equation mixes such degrees of freedom.

\section{EXAMPLES}

In this section we present some examples of metrics with and without flux.

\section{A. The case $V=m, F=0$}

When $V=m, F=0$, it is possible to use solutions of the massless Dirac equation on $\hat{M}$ to build solutions of the Dirac equation with mass on $\mathcal{M}$. It is also possible to perform the inverse operation and lift solutions from $\mathcal{M}$ to $\hat{M}$. There is a number of known examples of nontrivial spacetimes with Euclidean signature that admit CKY tensors. Following Ref. [43] we can take $\mathcal{M}$ to be the sphere $S^{n}$, which admits both KY and CCKY forms; Sasakian manifolds, which admit a rank 2 CCKY form, as, for example, $S^{1}$ bundles over Kähler manifolds; Kähler and nearly Kähler spaces, which admit a rank $2 \mathrm{CKY}$ form; and $G_{2}$ and weak- $G_{2}$ manifolds. There is also a classification of compact manifolds of Riemannian signature that admit special KY forms (for the definition of special KY forms we refer the reader to Ref. [43]), and these are Sasakian, nearly Kähler, and weak- $G_{2}$ manifolds. We can also consider $\mathcal{M}$ to be a Riemannian space admitting Killing spinors, along the lines of Ref. [44], as Killing spinors generate a tower of KY and CCKY forms. In particular, we can consider all spaces of special holonomy [Calabi-Yau, hyperKähler, $G_{2}$, Spin(7)]; maximally symmetric spaces; compact spaces with positive curvature the cone over which is irreducible, which include the already mentioned 3-Sasaki manifolds when $n=4 m-1, m$ is an integer, with hyperKähler cone, Sasaki-Einstein manifolds when $n=4 m \pm 1$ with Calabi-Yau cone, the already mentioned almost Kähler case when $n=6$, which has $G_{2}$ cone, and weak- $G_{2}$, which has $\operatorname{Spin}(7)$ cone. There are also noncompact spaces with negative curvature, in this case either the hyperbolic space $H^{n}$ or a warped product $M=N \times \mathbb{R}$ with metric $d s^{2}=e^{\mu y} d s_{N}^{2}+d y^{2}$, where $\mu \in \mathbb{R} 0$ and $N$ is 
a complete, connected spin manifold that admits nontrivial parallel spinors.

Another nontrivial example of Riemannian metric with CKY forms is to consider $\mathcal{M}$ to be a manifold with the canonical metric, which as shown in Refs. [32,67] is the most general metric that admits a principal conformal Killing-Yano form, that is, a rank 2 nondegenerate CCKY form. This metric depends on a set of one-variable functions, and when these are chosen so that the metric satisfies the Einstein vacuum equations with cosmological constant, then one obtains the (Wick rotated) Kerr-NUT-(A)dS metric of Ref. [22]. The canonical metric in $n=2 N+\epsilon$ dimensions, where $\epsilon=0,1$, admits $N+\epsilon$ Killing vectors and $N$ CCKY forms of even rank. By the results of Sec. III it is possible to lift all of these tensors to KY and CCKY tensors on $\hat{M}$. The metric $\hat{g}$ on $\hat{\mathcal{M}}$ also has the two Killing vectors $\partial_{v}, \partial_{t}$. The Dirac equation on $\mathcal{M}$ admits separation of variables due to a complete set of mutually commuting operators, one of which is the Dirac operator $[15,16]$. Then if $\chi_{1}$ is a solution of the massive Dirac equation on $\mathcal{M}$, one can upgrade it to a solution of the massless Dirac equation on $\hat{M}$ by setting $\chi_{2}=\chi_{1}$.

\section{B. The case $V$ a central potential, $F=0$}

Consider a flat Riemannian space $\mathcal{M}=\mathbb{R}^{n}$. Suppose the electromagnetic field is zero, $F=0$, and the potential $V$ is central, $V=V(r)$, with $r^{2}=x_{\mu} x^{\mu}$. Then we can consider the rank $n-1 \mathrm{KY}$ tensor

$$
f_{\lambda_{1} \ldots \lambda_{n-1}}=x^{\mu} \epsilon_{\mu \lambda_{1} \ldots \lambda_{n-1}},
$$

built using the totally antisymmetric Levi-Civita $n$ form $\epsilon_{\lambda_{1} \ldots \lambda_{n}}$. There is a conserved tensor associated with any geodesic on $\mathcal{M}$, which is given by $C_{\lambda_{1} \lambda_{n-2}}=\dot{x}^{\mu} f_{\mu \lambda_{1} \ldots \lambda_{n-2}}$. When $n=3$, the conserved quantity is proportional to the angular momentum, and therefore this example includes cases such as the Kepler problem or the harmonic oscillator; for $d>3$ we can think of this as a generalization of angular momentum. That the quantity is conserved can be seen by direct differentiation, noticing that the geodesic equation implies that $\ddot{x}$ is proportional to $\vec{\nabla} V=\frac{d V}{d r} \frac{\vec{x}}{r}$, and therefore

$$
\dot{C}_{\lambda_{1} \ldots \lambda_{n-2}} \propto \frac{d V}{d r} \frac{x^{\mu}}{r} x^{\nu} \epsilon_{\mu \nu \lambda_{1} \ldots \lambda_{n-2}}=0 .
$$

This condition is equivalent to $\left.d V^{\sharp}\right\lrcorner f=0$, which is the same as Eq. (3.14) and guarantees that we can promote $f$ to a KY tensor on the Eisenhart-Duval lift manifold $\hat{M}$, and build conserved tensors for the null geodesic motion on $\hat{M}$.

\section{The case $V \neq 0, F \neq 0$}

We present here a very simple example with the intent of showing that the projection (4.20) does indeed admit nontrivial solutions. From the construction of this example, however, it will become clear that interesting nontrivial solutions will in general require more effort.

We take $\mathcal{M}$ to be three-dimensional flat Euclidean space. The Gamma matrices are $\Gamma_{1}=\sigma_{1}, \Gamma_{2}=\sigma_{2}, \Gamma_{3}=$ $\sigma_{3}$, and the Pauli matrices. We consider a magnetic field $F=\phi(x) \sigma_{1} \sigma_{2}=i \phi \sigma_{3}$, and as an ansatz for the spinor $\chi_{2}$ we take the spinor $\chi_{2}=(1,0)$. The projection becomes

$$
i V \chi_{1}=i\left(V+\frac{e \phi}{4 m}\right) \chi_{2}=i V \tilde{\phi} \chi_{2},
$$

where we have defined the function

$$
\tilde{\phi}=1+\frac{e \phi}{4 m V} .
$$

The Dirac equation on $\hat{M}$ gives

$$
D \chi_{1}+i V \chi_{1}=0, \quad D \chi_{2}=i m \chi_{1} .
$$

One can check by direct substitution that these two coupled equations are compatible with the projection only if

$$
(i V+d(\ln \tilde{\phi})) \chi_{2}=-i m \tilde{\phi} \chi_{2} .
$$

Then it must be $\tilde{\phi}=\tilde{\phi}\left(x_{3}\right)$ in which case the equation above becomes an equation for the function $\tilde{\phi}$ :

$$
i V+d(\ln \tilde{\phi})=-i m \tilde{\phi} .
$$

But $V$ and $\tilde{\phi}$ are both real, and therefore it must be that $\tilde{\phi}$ is constant and that $V=-m \tilde{\phi}$. Then also $V$ and the magnetic field are constant. The Dirac equation on $\hat{M}$ reduces to the single equation

$$
D \chi_{2}=i m \tilde{\phi} \chi_{2},
$$

where $m \tilde{\phi}$ plays the role of an effective mass and a solution is given by

$$
\left(\begin{array}{c}
e^{i m \tilde{\phi} x_{3}} \\
0
\end{array}\right)
$$

\section{DISCUSSION AND CONCLUSIONS}

In this paper we have studied the Eisenhart-Duval lift from the point of view of hidden symmetries of the Dirac equation and have gained insight on the relationship between the procedure of lift/oxidation, its inverse procedure of reduction, and the symmetry operators of the Dirac equation with flux.

We have shown how the massless Dirac equation on $\hat{M}$ can be dimensionally reduced to a nonrelativistic LévyLeblond equation on $\mathcal{M}$. We have also discussed those cases where it is possible to obtain on $\mathcal{M}$ again a Dirac equation. When $V=-m$ and $F=0$, it is always possible to obtain by reduction the massive Dirac equation on $\mathcal{M}$ and to lift the hidden symmetries on $\mathcal{M}$ to hidden symmetries on $\hat{\mathcal{M}}$. However, for generic fluxes the Dirac equations in lower and higher dimension are not equivalent 
systems when considered from the point of view of their dynamics in phase space, since each theory can have hidden symmetries that are not present in the other. We have shown an example where the impossibility to lift some CKY tensors in the absence of flux is related to the doubling of the spinor degrees of freedom in higher dimension and the way that the higher dimensional Dirac equation mixes such degrees of freedom, and examples where the impossibility to dimensionally reduce instead is related to the fact that symmetries of the Dirac equation with flux on $\mathcal{M}$ present anomalies and not all $\mathrm{KY}$ and CCKY tensors are allowed. Whenever it is possible to either lift or dimensionally reduce the symmetry operators, then we find a geometrical relation between the symmetry operators of the two theories, one with flux and the other without. The situation is different from what happens analyzing the Eisenhart-Duval lift for a scalar particle: in that case all hidden symmetries of the lower dimensional theory can be lifted to hidden symmetries in higher dimension.

A by-product of this analysis is that we can build new Lorentzian metrics with $\mathrm{KY}$ and CCKY tensors, by lifting KY and CCKY tensors defined on Riemannian metrics. We also have presented a classification of the most general CKY tensor for the Eisenhart-Duval metric in terms of a set of equations for forms on the base manifold, both in the $v, t$ independent case and in the general one.

There is a number of questions left open in this work. One of these is the following: what is the actual form of generic solutions of the CKY equations on $\hat{\mathcal{M}}$ in terms of forms on $\mathcal{M}$, and do these solutions give any nontrivial generalization of the CKY equations? Another one is related to supergravity solutions: it is known how spacetimes with a null covariantly constant (Killing) vector can provide supersymmetric solutions of supergravity theories. It would be interesting to know if the present construction can be used in the context of supergravity to either provide new solutions or discuss existing ones in terms of hidden symmetries. Also it would be interesting to know if the tools used in the present analysis can also be used to study the more general class of Kundt spacetimes; see Ref. [68] for a recent discussion of their role in supergravity and string/ $M$-theory.

\section{ACKNOWLEDGMENTS}

The author would like to thank G.W. Gibbons for pointing out to him some of the open questions related to the Eisenhart-Duval lift and CKY tensors; P. Horváthy for numerous suggestions; and D. Kubizňák and C. M. Warnick for reading the manuscript and useful discussions. Part of this work has been done during the author's stay at ICTP-SAIFR in São Paulo; hospitality there and financial support are kindly acknowledged. The author is partially funded by Fapemig under Project No. CEX APQ 2324-11.

\section{APPENDIX A: EISENHART-DUVAL METRIC}

The nonzero Christoffel symbols for the EisenhartDuval lift metric (3.4) are

$$
\begin{array}{rlrl}
\hat{\Gamma}_{t t}^{v} & =-\frac{e}{m^{2}} A_{\lambda} \partial^{\lambda} V, & \hat{\Gamma}_{\mu t}^{v}=\frac{1}{2}\left(\frac{e^{2}}{m^{2}} A_{\lambda} F_{\mu}^{\lambda}-\frac{2}{m} \partial_{\mu} V\right), \\
\hat{\Gamma}_{\mu \nu}^{v}=\frac{e}{m} \nabla_{(\mu} A_{\nu)}, & \hat{\Gamma}_{t t}^{\lambda}=\frac{1}{m} \partial^{\lambda} V, \\
\hat{\Gamma}_{\mu t}^{\lambda}=-\frac{e}{2 m} F_{\mu}^{\lambda}, & \hat{\Gamma}_{\mu \nu}^{\lambda}=\Gamma_{\mu \nu}^{\lambda} .
\end{array}
$$

The covariantly constant Killing vector is $\partial_{v}$ and its associated 1 form is $\left(\partial_{v}\right)^{b}=d t$. A convenient choice for the vielbeins is

$$
\begin{aligned}
\hat{e}^{+} & =d t, \\
\hat{e}^{-} & =d v-\frac{V}{m} d t+\frac{e}{m} A_{\mu} d x^{\mu}, \\
\hat{e}^{a} & =e^{a},
\end{aligned}
$$

where $\left\{e^{a}, a=1, \ldots, n\right\}$ is a set of vielbeins for $\mathcal{M}$, and the $(n+2)$-dimensional Minkowski metric $\hat{\eta}_{A B}$ has the following nonzero entries: $\hat{\eta}_{+-}=\hat{\eta}_{-+}=1, \hat{\eta}_{a b}=\eta_{a b}$. The corresponding dual basis vectors are

$$
\begin{aligned}
& \left(\hat{e}^{+}\right)^{\#}=\hat{X}^{+}=\partial_{v}, \quad\left(\hat{e}^{-}\right)^{\#}=\hat{X}^{-}=\frac{V}{m} \partial_{v}+\partial_{t}, \\
& \left(\hat{e}^{a}\right)^{\#}=\hat{X}^{a}=-\frac{e}{m} A^{a} \partial_{v}+\left(e^{a}\right)^{\# g} .
\end{aligned}
$$

These are related to the inverse vielbein $\hat{E}_{A}^{M}$ by

$$
\hat{E}_{A}=\hat{\eta}_{A B} \hat{X}^{B} .
$$

From Eq. (A2) we can read the nonzero coefficients of the spin-connection:

$$
\begin{aligned}
\hat{\omega}_{+a} & =-\frac{1}{m} \partial_{a} V \hat{e}^{+}+\frac{e}{2 m} F_{a b} e^{b}, \\
\hat{\omega}_{a b} & =\omega_{a b}-\frac{e}{2 m} F_{a b} \hat{e}^{+} .
\end{aligned}
$$

\section{APPENDIX B: HODGE DUALITY}

In this section we display identities for Hodge duality on $\mathcal{M}$; these are straightforwardly generalized to the Hodge duality on $\hat{\mathcal{M}}$ with the appropriate change of signature and number of dimensions.

The Levi-Civita tensor $\varepsilon$ is an antisymmetric $n$ form satisfying

$$
\varepsilon \wedge{ }_{n}^{\wedge} \varepsilon=s n !
$$

where $s$ is the product of signs in the metric signature. With this the Hodge dual of a homogeneous $p$ form $\omega$ can be defined as 


$$
* \omega=\frac{1}{p !} \omega \underset{p}{\wedge} \varepsilon
$$

It follows that

$$
\begin{aligned}
* * \omega & =s(-1)^{p(n-p)} \omega, \quad * 1=\varepsilon, \\
* \varepsilon & =s, \quad \varepsilon^{2}=(-1)^{\left[\frac{n}{2}\right]} s .
\end{aligned}
$$

The Hodge duality operation exchanges a wedge product into an interior product and vice versa: for any vector $X^{a}$ dual to a vielbein $e^{a}$ it holds that

$$
\begin{aligned}
& \left.*\left(e^{a} \wedge \omega\right)=(-1)^{p} X^{a}\right\lrcorner(* \omega), \\
& \left.*\left(X^{a}\right\lrcorner \omega\right)=(-1)^{p+1} e^{a} \wedge(* \omega) .
\end{aligned}
$$

Also it transforms a contracted wedge product into a contracted wedge product. If $\alpha, \beta$ are homogeneous forms of degrees $p$ and $q$, then

$$
\begin{aligned}
*(\alpha \wedge \beta) & =\frac{k !}{(q-k) !}(-1)^{k(n-q)}(* \alpha) \underset{q-k}{\wedge} \beta \\
& =\frac{k !}{(p-k) !}(-1)^{p(q-k)} \alpha \underset{p-k}{\wedge}(* \beta) .
\end{aligned}
$$

\section{APPENDIX C: DIFFERENTIATION OF FORMS}

Consider a $p$ form $\hat{f}$ on $\hat{\mathcal{M}}$ that only has components on $\mathcal{M}$, according to

$$
\begin{aligned}
\hat{f} & =f_{M_{1} \ldots M_{p}}\left(v, t, x^{\mu}\right) \hat{e}^{M_{1}} \wedge \cdots \wedge \hat{e}^{M_{1}} \\
& =f_{\mu_{1} \ldots \mu_{p}}\left(v, t, x^{\mu}\right) e^{\mu_{1}} \wedge \cdots \wedge e^{\mu_{p}}
\end{aligned}
$$

When there is no $v, t$ dependence, then this yields a form $f$ on $\mathcal{M}$. We can explicitly calculate the components of the tensor $\hat{\nabla} \hat{f}$. We start with

$$
\begin{aligned}
\hat{\nabla}_{-} \hat{f}_{\mu_{1} \ldots \mu_{p}} & =\hat{E}_{-}^{M} \hat{\nabla}_{M} \hat{f}_{\mu_{1} \ldots \mu_{p}}=\hat{X}^{+M} \hat{\nabla}_{M} \hat{f}_{\mu_{1} \ldots \mu_{p}} \\
& =\hat{\nabla}_{v} \hat{f}_{\mu_{1} \ldots \mu_{p}}=\partial_{v} \hat{f}_{\mu_{1} \ldots \mu_{p}}+\left(\hat{\Gamma}_{v} \cdot f\right)_{\mu_{1} \ldots \mu_{p}} \\
& =\partial_{v} \hat{f}_{\mu_{1} \ldots \mu_{p}} .
\end{aligned}
$$

A similar calculation yields

$$
\left.\hat{\nabla}_{+} \hat{f}=\left(\frac{V}{m} \partial_{v}+\partial_{t}\right) \hat{f}+\frac{e}{2 m}(F \wedge \hat{f})-\frac{1}{m} \hat{e}^{+} \wedge\left(d V^{\#}\right\lrcorner \hat{f}\right),
$$

and

$$
\left.\left.\hat{\nabla}_{a} \hat{f}=-\frac{e}{m} A_{a} \partial_{v} \hat{f}+\nabla_{a} \hat{f}-\frac{e}{2 m} \hat{e}^{+} \wedge\left[\left(X_{a}\right\lrcorner F\right)\right\lrcorner \hat{f}\right] .
$$

With this we are able to calculate

$$
\begin{aligned}
\hat{d} \hat{f}= & \hat{e}^{A} \wedge \hat{\nabla}_{A} \hat{f} \\
= & e^{+} \wedge\left(\frac{V}{m} \partial_{v}+\partial_{t}\right) \hat{f}+e^{-} \wedge \partial_{v} \hat{f} \\
& +\left(-\frac{e}{m} A \wedge \partial_{v} \hat{f}+d \hat{f}\right),
\end{aligned}
$$

and

$$
\begin{aligned}
\hat{\delta} \hat{f} & \left.=-\left(\hat{e}^{A}\right)^{\#}\right\lrcorner \hat{\nabla}, \hat{f} \\
& \left.=\frac{e}{m} A^{\#}\right\lrcorner \partial_{v} \hat{f}+\delta \hat{f}+\frac{e}{2 m} e^{+} \wedge\left(F_{2} \hat{f}\right) .
\end{aligned}
$$

Analogous relations for the forms $\hat{e}^{+}$and $\hat{e}^{-}$are

$\hat{\nabla}_{A} \hat{e}^{+}=0 \quad \forall M=+,-, a, \quad \hat{\nabla}_{-} e^{-}=0$,

$\left.\hat{\nabla}_{+} e^{-}=\frac{1}{m} d V, \quad \hat{\nabla}_{a} e^{-}=\frac{e}{2 m} X_{a}\right\lrcorner F$.

\section{APPENDIX D: THE FULL CKY EQUATION}

From the $\hat{\nabla}_{-}$equation we get four identities:

$(--) \partial_{v} \rho^{-}=0$,

$$
\begin{aligned}
(-+) & \frac{p}{p+1} \partial_{v} \rho^{+} \\
= & \frac{1}{p+1}\left(-\left(\frac{V}{m} \partial_{v}+\partial_{t}\right) \rho^{-}+\frac{e}{m} A \wedge \partial_{v} g-d g\right) \\
& -\frac{1}{n+3-p}\left(\frac{e}{m} A^{\#}\right\lrcorner \partial_{v} f+\delta f-\partial_{v} \rho^{+} \\
& \left.-\left(\frac{V}{m} \partial_{v}+\partial_{t}\right) \rho^{-}-\frac{e}{2 m} F \wedge \rho^{-}\right), \\
(- \pm) & \left.(n+2-p) \partial_{v} g=\left(\delta \rho^{-}+\frac{e}{m} A^{\#}\right\lrcorner \partial_{v} \rho^{-}+\partial_{v} g\right), \\
(-a) & \frac{p}{p+1} \partial_{v} f=\frac{1}{p+1}\left(\frac{e}{m} A \wedge \partial_{v} \rho^{-}-d \rho^{-}\right) .
\end{aligned}
$$

We can think of these as equations for the partial $v$ derivatives of the forms $f, \rho^{ \pm}$, and $g$. Then from $\hat{\nabla}_{+}$ 


$$
\begin{aligned}
& (+-) \frac{p}{p+1}\left(\frac{V}{m} \partial_{v}+\partial_{t}\right) \rho^{-}+\frac{e}{2 m} F \wedge \rho_{1}^{-}=\frac{1}{p+1}\left(-\partial_{v} \rho^{+}-\frac{e}{m} A \wedge \partial_{v} g+d g\right)-\frac{1}{n+3-p} \\
& \left.\quad \times\left(\delta f+\frac{e}{m} A\right\lrcorner \partial_{v} f-\partial_{v} \rho^{+}-\left(\frac{V}{m} \partial_{v}+\partial_{t}\right) \rho^{-}-\frac{e}{2 m} F \wedge \rho_{1}^{-}\right), \\
& \left.(++)-\frac{d V^{\#}}{m}\right\lrcorner f+\left(\frac{V}{m} \partial_{v}+\partial_{t}\right) \rho^{+}+\frac{e}{2 m} F \wedge \rho^{+}+\frac{d V}{m} \wedge g=0, \\
& \left.\left.(+ \pm) \frac{n+2-p}{n+3-p}\left[\frac{d V^{\#}}{m}\right\lrcorner \rho^{-}+\left(\frac{V}{m} \partial_{v}+\partial_{t}\right) g\right]+\frac{e}{2 m} F \wedge g=-\frac{1}{n+3-p}\left(-\frac{e}{2 m} F \wedge f f+\frac{e}{m} A^{\#}\right\lrcorner \partial_{v} \rho^{+}+\delta \rho^{+}\right), \\
& (+a) \frac{p}{p+1}\left(\frac{V}{m} \partial_{v}+\partial_{t}\right) f+\frac{e}{2 m} F \wedge f+\frac{p}{p+1} \frac{d V}{m} \wedge \rho^{-}=\frac{1}{p+1}\left(\frac{e}{m} A \wedge \partial_{v} \rho^{+}-d \rho^{+}-\frac{e}{m} F \wedge g\right) .
\end{aligned}
$$

These are equations for the partial $t$ derivatives. Last, the $\hat{\nabla}_{a}$ equation gives

$$
\begin{aligned}
(a-) & \left.\left.-\frac{e}{m} A_{a} \partial_{v} \rho^{-}+\nabla_{a} \rho^{-}=\frac{X_{a}}{p+1}\right\lrcorner\left(-\partial_{v} f-\frac{e}{m} A \wedge \partial_{v} \rho^{-}+d \rho^{-}\right)-\frac{e_{a}}{n+3-p} \wedge\left(\delta \rho^{-}+\frac{e}{m} A^{\#}\right\lrcorner \partial_{v} \rho^{-}+\partial_{v} g\right), \\
(a+) & \left.\left.-\frac{e}{2 m}\left(X_{a}\right\lrcorner F\right) \wedge f+\nabla_{a} \rho^{+}-\frac{e}{m} A_{a} \partial_{v} \rho^{+}+\frac{e}{2 m}\left(X_{a}\right\lrcorner F\right) \wedge g \\
= & \left.\frac{X_{a}}{p+1}\right\lrcorner\left[-\left(\frac{V}{m} \partial_{v}+\partial_{t}\right) f-\frac{e}{m} A \wedge \partial_{v} \rho^{+}+d \rho^{+}-\frac{d V}{m} \wedge \rho^{-}+\frac{e}{m} F \wedge g\right] \\
& \left.\left.-\frac{e_{a}}{n+3-p} \wedge\left[-\frac{e}{2 m} F \wedge f+\frac{e}{m} A^{\#}\right\lrcorner \partial_{v} \rho^{+}+\delta \rho^{+}-\frac{d V^{\#}}{m}\right\lrcorner \rho^{-}\right], \\
(a \pm) & \left.\nabla_{a} g+\frac{e}{2 m}\left(X_{a}\right\lrcorner F\right) \wedge \rho_{1}^{-}-\frac{e}{m} A_{a} \partial_{v} g \\
= & \left.\left.\frac{X_{a}}{p+1}\right\lrcorner\left(-\partial_{v} \rho^{+}+\left(\frac{V}{m} \partial_{v}+\partial_{t}\right) \rho^{-}-\frac{e}{m} A \wedge \partial_{v} g+d g\right)-\frac{e_{a}}{n+3-p} \wedge\left(\delta g+\frac{e}{m} A^{\#}\right\lrcorner \partial_{v} g+\frac{e}{2 m} F \wedge \rho^{-}\right), \\
(a b)= & \left.\frac{e}{m} A_{a} \partial_{v} f+\nabla_{a} f+\frac{e}{2 m}\left(X_{a}\right\lrcorner F\right) \wedge \rho^{-} \\
= & \left.\frac{X_{a}}{p+1}\right\lrcorner\left(-\frac{e}{m} A \wedge \partial_{v} f+d f+\frac{e}{m} F \wedge \rho^{-}\right) \\
& \left.-\frac{e_{a}}{n+3-p} \wedge\left(\delta f+\frac{e}{m} A^{\#}\right\lrcorner \partial_{v} f-\partial_{v} \rho^{+}-\left(\frac{V}{m} \partial_{v}+\partial_{t}\right) \rho^{-}-\frac{e}{2 m} F \wedge \rho_{1}^{-}\right) .
\end{aligned}
$$

Further simplifications can be obtained taking each of the four $\hat{\nabla}_{a}$ equations, and calculating the product $e^{a} \wedge$ and $\left.X^{a}\right\lrcorner$, summing over $a$. This gives equations for the differential and codifferential of $f, \rho^{ \pm}$, and $g$ that can be put back in the other CKY equations.

[1] L. P. Eisenhart, Ann. Math. 30, 591 (1928).

[2] C. Duval, G. Burdet, H. P. Künzle, and M. Perrin, Phys. Rev. D 31, 1841 (1985).

[3] C. Duval, G. W. Gibbons, and P. Horváthy, Phys. Rev. D 43, 3907 (1991).

[4] P. M. Zhang, G. W. Gibbons, and P. A. Horváthy, Phys. Rev. D 85, 045031 (2012).

[5] P. A. Horváthy and P. Zhang, Phys. Rep. 481, 83 (2009).

[6] S. Benenti, J. Math. Phys. (N.Y.) 38, 6578 (1997).

[7] I. M. Benn, J. Math. Phys. (N.Y.) 47, 022903 (2006).

[8] G. W. Gibbons and C. N. Pope, Ann. Phys. (N.Y.) 326, 1760 (2011).

[9] L. N. Mazzoni and L. Casetti, Phys. Rev. E 77, 051917 (2008).
[10] L. Casetti and A. Macchi, Phys. Rev. E 55, 2539 (1997).

[11] M. Cerruti-Sola and M. Pettini, Phys. Rev. E 51, 53 (1995).

[12] D. N. Page, D. Kubizňák, M. Vasudevan, and P. Krtouš, Phys. Rev. Lett. 98, 061102 (2007).

[13] V.P. Frolov, P. Krtouš, and D. Kubizňák, J. High Energy Phys.02 (2007) 005.

[14] A. Sergyeyev and P. Krtouš, Phys. Rev. D 77, 044033 (2008).

[15] T. Oota and Y. Yasui, Phys. Lett. B 659, 688 (2008).

[16] M. Cariglia, P. Krtouš, and D. Kubizňák, Phys. Rev. D 84, 024008 (2011). 
[17] K. Murata and J. Soda, Classical Quantum Gravity 25, 035006 (2008).

[18] H. Kodama and A. Ishibashi, Prog. Theor. Phys. 110, 701 (2003).

[19] H. K. Kunduri, J. Lucietti, and H. S. Reall, Phys. Rev. D 74, 084021 (2006).

[20] T. Oota and Y. Yasui, Int. J. Mod. Phys. A 25, 3055 (2010).

[21] O. Dias, P. Figueras, R. Monteiro, H. Reall, and J. Santos, J. High Energy Phys. 05 (2010) 076.

[22] W. Chen, H. Lü, and C.N. Pope, Classical Quantum Gravity 23, 5323 (2006).

[23] D. Kubizňák and V. P. Frolov, Classical Quantum Gravity 24, F1 (2007).

[24] D. Kubizñák and V. P. Frolov, Classical Quantum Gravity 25, 154005 (2008).

[25] M. Cariglia and D. Kubizňák, Phys. Rev. Lett. 108, 051104 (2012).

[26] D. Kubizňák, Ph.D. thesis, University of Alberta, 2008.

[27] T. Houri and Y. Yasui, Prog. Theor. Phys. Suppl. 189, 126 (2011).

[28] O. P. Santillan, J. Math. Phys. (N.Y.) 53, 043509 (2012).

[29] G. W. Gibbons, T. Houri, D. Kubizńák, and C.M. Warnick, Phys. Lett. B 700, 68 (2011).

[30] G. W. Gibbons and C. Rugina, J. Math. Phys. (N.Y.) 52, 122901 (2011).

[31] A. Galajinsky, Phys. Rev. D 85, 085002 (2012).

[32] V. P. Frolov, P. Krtouš, and D. Kubizňák, Phys. Rev. D 78, 064022 (2008).

[33] T. Houri, T. Oota, and Y. Yasui, Classical Quantum Gravity 26, 045015 (2009).

[34] H. Ahmedov and A. N. Aliev, Phys. Lett. B 679, 396 (2009).

[35] T. Houri, D. Kubizňák, C. M. Warnick, and Y. Yasui, arXiv:1203.0393.

[36] I. M. Benn and P. Charlton, Classical Quantum Gravity 14, 1037 (1997).

[37] I. M. Benn and J. Kress, Classical Quantum Gravity 21, 427 (2004).

[38] P. Krtouš, D. Kubizňák, and C. Warnick, Nucl. Phys. B844, 185 (2011).

[39] E. R. de Celis and O.P. Santillan, arXiv:1205.3256.

[40] B. Carter, Phys. Rev. D 16, 3395 (1977).

[41] M. Cariglia, P. Krtouš, and D. Kubizňák, Phys. Rev. D 84, 024004 (2011).

[42] G. W. Gibbons, R. H. Rietdijk, and J. W. van Holten, Nucl. Phys. B404, 42 (1993).
[43] U. Semmelmann, Math. Z. 245, 503 (2003).

[44] M. Cariglia, Classical Quantum Gravity 21, 1051 (2004).

[45] T. Houri, D. Kubizňák, C. M. Warnick, and Y. Yasui, arXiv:1203.0393.

[46] T. Houri, D. Kubizňák, C. M. Warnick, and Y. Yasui, Classical Quantum Gravity 27, 185019 (2010).

[47] G. Papadopoulos, Classical Quantum Gravity 25, 105016 (2008).

[48] G. Papadopoulos, arXiv:1111.6744.

[49] O.P. Santillan, arXiv:1108.0149.

[50] F. Correa, V. Jakubsky, and M. S. Plyushchay, Ann. Phys. (N.Y.) 324, 1078 (2009).

[51] V. Jakubsky, L. M. Nieto, and M. S. Plyushchay, Phys. Rev. D 83, 047702 (2011).

[52] R. Jackiw and N. S. Manton, Ann. Phys. (N.Y.) 127, 257 (1980).

[53] C. Duval, P. Horváthy, Ann. Phys. (N.Y.) 142, 10 (1982).

[54] J. W. van Holten, Phys. Rev. D 75, 025027 (2007).

[55] P. Horváthy and J. P. Ngome, Phys. Rev. D 79, 127701 (2009).

[56] M. Visinescu, Mod. Phys. Lett. A 26, 2719 (2011).

[57] J. P. Ngome, J. Math. Phys. (N.Y.) 50, 122901 (2009).

[58] G. W. Gibbons and P. J. Ruback, Phys. Lett. B 188, 226 (1987).

[59] L. Fehér and P. A. Horváthy, Phys. Lett. B 201, 481 (1988).

[60] J. W. van Holten, Phys. Lett. B 342, 47 (1995).

[61] D. Vaman and M. Visinescu, Phys. Rev. D 57, 3790 (1998).

[62] D. Baleanu and S. Codoban, Gen. Relativ. Gravit. 31, 497 (1999).

[63] M. Visinescu, in Noncommutative Structures in Mathematics and Physics, edited by S. Duplij and J. Wess (Kluwer Academic Publishers, Dordrect, 2001), Vol. 22, p.441; J. Phys. A 33, 4383 (2000).

[64] J. M. Lévy-Leblond, Commun. Math. Phys. 6, 286 (1967).

[65] C. Duval, P. A. Horváthy, and L. Palla, Ann. Phys. (N.Y.) 249, 265 (1996).

[66] G. W. Gibbons and D. L. Wiltshire, Ann. Phys. (N.Y.) 167, 201 (1986).

[67] T. Houri, T. Oota, and Y. Yasui, Phys. Lett. B 656, 214 (2007).

[68] J. Brannlund, A. Coley, and S. Hervik, Classical Quantum Gravity 25, 195007 (2008). 